\title{
Nonlinear Dirac equation solitary waves in external fields
}

\author{
Franz G. Mertens, ${ }^{1, *}$ Niurka R. Quintero, ${ }^{2, \dagger}$ Fred Cooper, ${ }^{3,4, \ddagger}$ Avinash Khare, ${ }^{5,8}$ and Avadh Saxena ${ }^{4, \mid}$ \\ ${ }^{1}$ Physikalisches Institut, Universität Bayreuth, D-95440 Bayreuth, Germany \\ ${ }^{2}$ IMUS and Departamento de Fisica Aplicada I, E.P.S. Universidad de Sevilla, 41011 Sevilla, Spain \\ ${ }^{3}$ Santa Fe Institute, Santa Fe, New Mexico 87501, USA \\ ${ }^{4}$ Theoretical Division and Center for Nonlinear Studies, Los Alamos National Laboratory, Los Alamos, New Mexico 87545, USA \\ ${ }^{5}$ Indian Institute of Science Education and Research, Pune 411021, India
}

(Received 13 August 2012; published 24 October 2012)

\begin{abstract}
We consider nonlinear Dirac equations (NLDE's) in the $1+1$ dimension with scalar-scalar self-interaction $\frac{g^{2}}{\kappa+1}(\bar{\Psi} \Psi)^{\kappa+1}$ in the presence of various external electromagnetic fields. We find exact solutions for special external fields and we study the behavior of solitary-wave solutions to the NLDE in the presence of a wide variety of fields in a variational approximation depending on collective coordinates which allows the position, width, and phase of these waves to vary in time. We find that in this approximation the position $q(t)$ of the center of the solitary wave obeys the usual behavior of a relativistic point particle in an external field. For time-independent external fields, we find that the energy of the solitary wave is conserved but not the momentum, which becomes a function of time. We postulate that, similarly to the nonlinear Schrödinger equation (NLSE), a sufficient dynamical condition for instability to arise is that $d P(t) / d \dot{q}(t)<0$. Here $P(t)$ is the momentum of the solitary wave, and $\dot{q}$ is the velocity of the center of the wave in the collective coordinate approximation. We found for our choices of external potentials that we always have $d P(t) / d \dot{q}(t)>0$, so, when instabilities do occur, they are due to a different source. We investigate the accuracy of our variational approximation using numerical simulations of the NLDE and find that, when the forcing term is small and we are in a regime where the solitary wave is stable, that the behavior of the solutions of the collective coordinate equations agrees very well with the numerical simulations. We found that the time evolution of the collective coordinates of the solitary wave in our numerical simulations, namely the position of the average charge density and the momentum of the solitary wave, provide good indicators for when the solitary wave first becomes unstable. When these variables stop being smooth functions of time $(t)$, then the solitary wave starts to distort in shape.
\end{abstract}

DOI: 10.1103/PhysRevE.86.046602

PACS number(s): 05.45.Yv, 03.70.+k, 11.10.Lm

\section{INTRODUCTION}

Classical solutions of nonlinear field equations have a long history as a model of extended particles [1-3]. In 1970, Soler [3] proposed that the self-interacting 4-Fermi theory was an interesting model for extended fermions. Later, Strauss and Vasquez [4] were able to study the stability of this model under dilatation and found the domain of stability for the Soler solutions. Solitary waves in the $1+1$ dimensional nonlinear Dirac equation (NLDE) have been studied $[5,6]$ in the past in the case of massive Gross-Neveu [7] (with $N=1$, i.e., just one localized fermion) and massive Thirring [8] models. In those studies, it was found that these equations have solitary-wave solutions for both scalar-scalar (S-S) and vector-vector $(\mathrm{V}-\mathrm{V})$ interactions. The interaction between solitary waves of different initial charge was studied in detail for the S-S case in the work of Alvarez and Carreras [9] by Lorentz boosting the static solutions and allowing them to scatter. Recently, we extended the solutions previously found to a more general interaction of the form $\frac{g^{2}}{\kappa+1}(\bar{\Psi} \Psi)^{\kappa+1}$ [10]. For the nonrelativistic limit of the NLDE, namely the nonlinear Schrödinger equation (NLSE), there have been

\footnotetext{
*franzgmertens@gmail.com

†niurka@us.es

¥cooper@santafe.edu

§khare@iiserpune.ac.in

lavadh@lanl.gov
}

recent studies of the behavior of the forced NLSE. Using a collective coordinate (CC) theory, the authors found [11-14] that a sufficient dynamical condition for instability to arise is that $d p(t) / d v<0$. Here $p(t)$ is the normalized canonical momentum $p(t)=\frac{1}{M(t)} \frac{\partial L}{\partial \dot{q}}, M(t)=\int d x \Psi^{\star}(x, t) \Psi(x, t)$ is the mass, and $\dot{q}(t)=v(t)$ is the velocity of the solitary wave.

One of the points we will investigate in the paper is whether this dynamical stability criterion is also valid for the NLDE. There has been recent interest in the stability of NLDE with higher-order nonlinearity [15]. Comech (private communication) has been able to prove that for $\kappa=1$, the Vakhitov-Kolokolov [16] criterion guarantees linear stability in the nonrelativistic regime of the NLDE equation for solutions of the form (in the rest frame) $\Psi(x, t)=\psi(x) e^{-i \omega t}$, where $\omega$ is less than but approximately equal to the mass parameter $m$ in the Dirac equation. He was also able to show linear instability in the same nonrelativistic regime for $\kappa \geqslant 3$. This is the first rigorous result for the Dirac equation, but it only applies in the nonrelativistic regime. Here we want to understand if we can determine in the relativistic regime for what values of $\omega$ do the solitary waves become unstable, with and without forcing terms, even when they are stable in the nonrelativistic regime. What we find is that when the solitary waves are only metastable for the unforced problem, the critical time for the solitary wave to become unstable in the forced problem for weak forcing is similar to the critical time in the unforced problem. When the solitary wave maintains its basic shape, the CC equations give a good description of the actual time evolution at all times. This is true for weak 
ramp potentials, harmonic potentials, and spatially periodic potentials, when $\omega>\omega_{c}$, and $\omega_{c}$ is the critical value above which the unforced solitary wave is stable. The collective coordinates $q(t)$ and $P(t)$, the position and momentum of the solitary wave, are "smooth" functions of $t$ for the external potentials we have chosen. Their counterparts in the numerical simulation are the first moment of the charge density and the total momentum of the numerical solution. When the numerical evolution of these counterparts to the collective coordinates start deviating from their $\mathrm{CC}$ values, this is a signal that the shape of the solitary wave is beginning to change. This usually rapidly develops into non-smooth behavior of $q(t)$ and $P(t)$ in the numerical solution. This is how we determine the onset of the instability time $t_{c}$ for the forced NLDE solitary wave. Unfortunately, for the potentials we study, we always obtain $d p / d \dot{q}>0$, which fulfills a necessary condition for stability. Thus, this criterion does not yield a prediction of the instabilities.

This paper is organized as follows: In Sec. II, we review the known exact solutions for the unforced NLDE and discuss the conservation laws that govern their behavior. In Sec. III we extend Bogolubsky's discussion [17] of the stability of these solitary-wave solutions to changes in the frequency $\omega$ for arbitrary nonlinearity parameter $\kappa$. In Sec. IV we consider the NLDE in external electromagnetic fields and find particular exact solitary-wave solutions and discuss the stability of these solutions. In Sec. V we introduce our variational method based on using for our variational wave functions the exact wave functions for the solitary waves of the unforced problem, with the position, width parameter, and phase of these solutions being promoted to collective coordinates depending on time. We write the relativistic equations for these collective coordinates which are similar to point particle relativistic dynamical equations. The potential the average position of the solitary wave sees is a particular average of the external potential weighted with the charge density. In Sec. VI we postulate our stability criterion for an arbitrary external potential based on just solving the $\mathrm{CC}$ equations. This condition is a sufficient condition for instability. In Sec. VII we examine and solve the collective coordinate (CC) equations for three types of potentials - a ramp potential, a harmonic potential, and a spatially periodic potential. We also compare the solution to the $\mathrm{CC}$ equations to the numerical simulation of the NLDE equation. We state our conclusions in Sec. VIII. In the appendix we discuss identities that are obeyed by the solutions in the rest frame.

\section{REVIEW OF EXACT SOLUTIONS TO THE NLDE}

In this section, we review the exact solutions to the NLDE, using the notation of Ref. [10]. We are interested in solitarywave solution of the NLDE given by

$$
\left(i \gamma^{\mu} \partial_{\mu}-m\right) \Psi+g^{2}(\bar{\Psi} \Psi)^{\kappa} \Psi=0 .
$$

These equations can be derived in a standard fashion from the Lagrangian density

$$
\mathcal{L}=\left(\frac{i}{2}\right)\left[\bar{\Psi} \gamma^{\mu} \partial_{\mu} \Psi-\partial_{\mu} \bar{\Psi} \gamma^{\mu} \Psi\right]-m \bar{\Psi} \Psi+\frac{g^{2}}{\kappa+1}(\bar{\Psi} \Psi)^{\kappa+1} .
$$

For solitary-wave solutions, the field $\Psi$ goes to zero at infinity. It is sufficient to go into the rest frame, since the theory is Lorentz invariant and the moving solution can be obtained by a Lorentz boost. In the rest frame we consider solutions of the form

$$
\Psi(x, t)=e^{-i \omega t} \psi(x) .
$$

We are interested in bound-state solutions that correspond to positive frequency $\omega \geqslant 0$ and which have energies in the rest frame less than the mass parameter $m$, i.e., $\omega<m$. In our previous paper [10], we chose the representation $\gamma_{0}=\sigma_{3}, i \gamma_{1}=\sigma_{1}$. Here, to make contact with the numerical simulations paper of Alvarez and Carreras [9] we choose instead $\gamma^{0}=\sigma_{3} ; \gamma^{1}=i \sigma_{2}$. Defining $A, B$ via

$$
\psi(x)=\left[\begin{array}{c}
A(x) \\
i B(x)
\end{array}\right]=R(x)\left(\begin{array}{c}
\cos \theta \\
i \sin \theta
\end{array}\right),
$$

we obtain the following equations for $A$ and $B$ :

$$
\begin{aligned}
& \frac{d A}{d x}+(m+\omega) B-g^{2}\left(A^{2}-B^{2}\right)^{\kappa} B=0, \\
& \frac{d B}{d x}+(m-\omega) A-g^{2}\left(A^{2}-B^{2}\right)^{\kappa} A=0 .
\end{aligned}
$$

A first integral of these equations can be obtained by realizing that from energy-momentum conservation we have

$\partial_{\mu} T^{\mu \nu}=0 ; T^{\mu \nu}=\frac{i}{2}\left[\bar{\Psi} \gamma^{\mu} \partial^{\nu} \Psi-\partial^{\nu} \bar{\Psi} \gamma^{\mu} \Psi\right]-g^{\mu \nu} \mathcal{L}$.

Thus, for stationary solutions,

$$
T^{10}=\text { const }, \quad T^{11}=\text { const. }
$$

Now, using (2.3), we obtain

$$
T^{11}=\omega \psi^{\dagger} \psi-m \bar{\psi} \psi+\mathcal{L}_{I} ; \mathcal{L}_{I}=\frac{g^{2}}{\kappa+1}(\bar{\psi} \psi)^{\kappa+1} .
$$

For solitary-wave solutions vanishing at infinity, the constant is zero and we get the useful first integral,

$$
T^{11}=\omega \psi^{\dagger} \psi-m \bar{\psi} \psi+\mathcal{L}_{I}=0 .
$$

Multiplying the equation of motion on the left by $\bar{\Psi}$ and using (2.3) we have that

$$
(\kappa+1) \mathcal{L}_{I}=-\omega \psi^{\dagger} \psi+m \bar{\psi} \psi-\bar{\psi} i \gamma^{1} \partial_{1} \psi .
$$

Therefore, we can rewrite $T^{11}=0$ as

$$
\omega \kappa \psi^{\dagger} \psi-m \kappa \bar{\psi} \psi-\bar{\psi} i \gamma^{1} \partial_{1} \psi=0
$$

For the Hamiltonian density we obtain

$$
\begin{aligned}
\mathcal{H} & =T^{00}=\frac{i}{2}\left[\bar{\Psi} \gamma^{1} \partial_{x} \Psi-\partial_{x} \bar{\Psi} \gamma^{1} \Psi\right]+m \bar{\Psi} \Psi-\mathcal{L}_{I} \\
& \equiv h_{1}+h_{2}-h_{3} .
\end{aligned}
$$

Each of the $h_{i}$ are positive definite. From Eqs. (2.9) and (2.10), one has the relationship

$$
\kappa \mathcal{L}_{I}=-\bar{\psi} i \gamma^{1} \partial_{x} \psi \text {. }
$$

From this, we have

$$
h_{3}=\frac{1}{\kappa} h_{1}
$$

and, in particular for $\kappa=1, \mathcal{H}=m \bar{\psi} \psi$. 
In terms of $R, \theta$ one has

$$
\bar{\psi} i \gamma_{1} \partial_{1} \psi=\psi^{\dagger} \psi \frac{d \theta}{d x} .
$$

This leads to the simple differential equation for $\theta$ for solitary waves,

$$
\frac{d \theta}{d x}=-\omega_{\kappa}+m_{\kappa} \cos 2 \theta ; \quad \omega_{\kappa} \equiv \kappa \omega ; \quad m_{\kappa}=\kappa m .
$$

The solution is (in this section and what follows we will choose the position of the solitary wave to be initially at $x_{0}=0$ )

$$
\theta(x)=\tan ^{-1}\left(\alpha \tanh \beta_{\kappa} x\right),
$$

where

$\alpha=\left(\frac{m_{\kappa}-\omega_{\kappa}}{m_{\kappa}+\omega_{\kappa}}\right)^{1 / 2}=\left(\frac{m-\omega}{m+\omega}\right)^{1 / 2}, \quad \beta_{\kappa}=\left(m_{\kappa}^{2}-\omega_{\kappa}^{2}\right)^{1 / 2}$.

Thus, we have

$$
\begin{aligned}
\tan \theta(x) & =\alpha \tanh \beta_{\kappa} x, \quad \sin ^{2} \theta(x)=\frac{(m-\omega) \sinh ^{2} \beta_{\kappa} x}{m \cosh 2 \beta_{\kappa} x+\omega} ; \\
\cos ^{2} \theta(x) & =\frac{(m+\omega) \cosh ^{2} \beta_{\kappa} x}{m \cosh 2 \beta_{\kappa} x+\omega},
\end{aligned}
$$

where we have used the identities

$$
\begin{aligned}
& 1+\alpha^{2} \tanh ^{2} \beta_{k} x=\left(\frac{m \cosh 2 \beta_{k} x+\omega}{m+\omega}\right) \operatorname{sech}^{2} \beta_{k} x, \\
& 1-\alpha^{2} \tanh ^{2} \beta_{k} x=\left(\frac{\omega \cosh 2 \beta_{k} x+m}{m+\omega}\right) \operatorname{sech}^{2} \beta_{k} x .
\end{aligned}
$$

Solving Eq. (2.9) for $R^{2}$ we obtain

$$
R^{2}=\left[\frac{(\kappa+1)(m \cos 2 \theta-\omega)}{g^{2}(\cos 2 \theta)^{\kappa+1}}\right]^{1 / \kappa}
$$

Now we have

$$
\frac{d \theta}{d x}=\frac{\beta_{\kappa}^{2}}{\omega_{\kappa}+m_{\kappa} \cosh 2 \beta_{\kappa} x}=-\omega_{\kappa}+m_{\kappa} \cos 2 \theta,
$$

where $\beta_{\kappa}=\sqrt{m_{\kappa}^{2}-\omega_{\kappa}^{2}}=\kappa \sqrt{m^{2}-\omega^{2}}$, so

$$
\cos 2 \theta=\frac{m_{\kappa}+\omega_{\kappa} \cosh 2 \beta_{\kappa} x}{\omega_{\kappa}+m_{\kappa} \cosh 2 \beta_{\kappa} x}=\frac{m+\omega \cosh 2 \beta_{\kappa} x}{\omega+m \cosh 2 \beta_{\kappa} x} .
$$

We can rewrite $R^{2}$ using the right-hand side of Eq. (2.22) as

$$
R^{2}=\left(\frac{\omega+m \cosh 2 \beta_{\kappa} x}{m+\omega \cosh 2 \beta_{\kappa} x}\right)\left[\frac{(\kappa+1) \beta_{\kappa}^{2}}{g^{2} \kappa^{2}\left(m+\omega \cosh 2 \beta_{\kappa} x\right)}\right]^{1 / \kappa} .
$$

Using the identities in Eq. (2.20), we obtain the alternative expression

$$
\begin{aligned}
R^{2}= & \left(\frac{1+\alpha^{2} \tanh ^{2} \beta_{\kappa} x}{1-\alpha^{2} \tanh ^{2} \beta_{\kappa} x}\right) \\
& \times\left[\frac{\operatorname{sech}^{2} \beta_{\kappa} x(\kappa+1) \beta_{\kappa}^{2}}{g^{2} \kappa^{2}(m+\omega)\left(1-\alpha^{2} \tanh ^{2} \beta_{\kappa} x\right)}\right]^{1 / \kappa} .
\end{aligned}
$$

In particular, for $\kappa=1$,

$$
R^{2}=\frac{2(m-\omega)}{g^{2}} \frac{\left(1+\alpha^{2} \tanh ^{2} \beta x\right)}{\left(1-\alpha^{2} \tanh ^{2} \beta x\right)^{2}} \operatorname{sech}^{2} \beta x
$$

and

$$
\begin{aligned}
& A^{2}=R^{2} \cos ^{2} \theta=\frac{2}{g^{2}} \frac{\left(m^{2}-\omega^{2}\right)(m+\omega) \cosh ^{2} \beta x}{(m+\omega \cosh 2 \beta x)^{2}}, \\
& B^{2}=R^{2} \sin ^{2} \theta=\frac{2}{g^{2}} \frac{\left(m^{2}-\omega^{2}\right)(m-\omega) \sinh ^{2} \beta x}{(m+\omega \cosh 2 \beta x)^{2}} .
\end{aligned}
$$

For arbitrary $\kappa$ we have

$$
\begin{aligned}
& A=\sqrt{\frac{(m+\omega) \cosh ^{2}(\kappa \beta x)}{m+\omega \cosh (2 \kappa \beta x)}}\left[\frac{(\kappa+1) \beta^{2}}{g^{2}(m+\omega \cosh (2 \kappa \beta x))}\right]^{\frac{1}{2 \kappa}}, \\
& B=\sqrt{\frac{(m-\omega) \sinh ^{2}(\kappa \beta x)}{m+\omega \cosh (2 \kappa \beta x)}}\left[\frac{(\kappa+1) \beta^{2}}{g^{2}(m+\omega \cosh (2 \kappa \beta x))}\right]^{\frac{1}{2 \kappa}} .
\end{aligned}
$$

Because of Lorentz invariance we can find the solution in a frame moving with velocity $v$ with respect to the rest frame. The Lorentz boost is given in terms of the rapidity variable $\eta$ as follows (here $c=1$ ):

$v=\tanh \eta ; \quad \gamma=\frac{1}{\sqrt{1-v^{2}}}=\cosh \eta ; \quad \sinh \eta=\frac{v}{\sqrt{1-v^{2}}}$.

In the moving frame, the transformation law for spinors tells us that

$$
\begin{aligned}
\Psi(x, t)= & \left(\begin{array}{lr}
\cosh (\eta / 2) & \sinh (\eta / 2) \\
\sinh (\eta / 2) & \cosh (\eta / 2)
\end{array}\right) \\
& \times\left(\begin{array}{l}
\Psi_{1}^{0}[\gamma(x-v t), \gamma(t-v x)] \\
\Psi_{2}^{0}[\gamma(x-v t), \gamma(t-v x)]
\end{array}\right),
\end{aligned}
$$

since

$$
\cosh (\eta / 2)=\sqrt{(1+\gamma) / 2} ; \quad \sinh (\eta / 2)=\sqrt{(\gamma-1) / 2} .
$$

This in component form,

$$
\begin{aligned}
& \Psi_{1}(x, t)=\left[\cosh (\eta / 2) A\left(x^{\prime}\right)+i \sinh (\eta / 2) B\left(x^{\prime}\right)\right] e^{-i \omega t^{\prime}}, \\
& \Psi_{2}(x, t)=\left[\sinh (\eta / 2) A\left(x^{\prime}\right)+i \cosh (\eta / 2) B\left(x^{\prime}\right)\right] e^{-i \omega t^{\prime}},
\end{aligned}
$$

where

$$
x^{\prime}=\gamma(x-v t) ; \quad t^{\prime}=\gamma(t-v x) .
$$

Note that $\cosh ^{2}(\eta / 2)+\sinh ^{2}(\eta / 2)=\cosh \eta=\gamma$.

\section{A. Conservation laws of the NLDE}

The Lagrangian is invariant under the transformation of phase $\Psi \rightarrow e^{i \Lambda} \Psi$, which by Noether's theorem leads to the conserved current,

$$
\partial_{\mu} j^{\mu}(x)=0 ; \quad j^{\mu}=\bar{\Psi} \gamma^{\mu} \Psi .
$$

This leads to charge conservation,

$$
Q=\int d x \Psi^{\dagger} \Psi
$$

which, for the solitary-wave solution, leads to

$$
Q=\int d x\left(A^{2}+B^{2}\right)=\frac{1}{\kappa \beta}\left[\frac{(\kappa+1) \beta^{2}}{g^{2}(m+\omega)}\right]^{1 / \kappa} I_{\kappa}\left(\alpha^{2}\right),
$$


where

$$
\begin{aligned}
I_{\kappa}\left(\alpha^{2}\right)= & \int_{-1}^{1} d y \frac{1+\alpha^{2} y^{2}}{\left(1-y^{2}\right)^{(\kappa-1) / \kappa}\left[1-\alpha^{2} y^{2}\right]^{(\kappa+1) / \kappa}} \\
= & B\left(\frac{1}{2}, \frac{1}{\kappa}\right){ }_{2} F_{1}\left(1+\frac{1}{\kappa}, \frac{1}{2}, \frac{1}{2}+\frac{1}{\kappa} ; \alpha^{2}\right) \\
& +\alpha^{2} B\left(\frac{3}{2}, \frac{1}{\kappa}\right){ }_{2} F_{1}\left(1+\frac{1}{\kappa}, \frac{3}{2}, \frac{3}{2}+\frac{1}{\kappa} ; \alpha^{2}\right),
\end{aligned}
$$

and ${ }_{2} F_{1}$ is a hypergeometric function. Here $B(x, k)$ denotes the $\beta$ function.

We also have energy-momentum conservation Eq. (2.6) leading to conservation of energy and momentum,

$$
E=\int T^{00} d x ; \quad P=\int T^{01} d x .
$$

Because of Lorentz invariance it is sufficient to calculate the energy-momentum tensor in the comoving frame $v=0$. The energy momentum tensor in an arbitrary frame is then given by

$$
T^{\mu \nu}=\Lambda_{\alpha}^{\mu} \Lambda_{\beta}^{\nu} T^{\alpha \beta} ; \quad \Lambda_{\alpha}^{\mu}=\left(\begin{array}{cc}
\cosh \eta & \sinh \eta \\
\sinh \eta & \cosh \eta
\end{array}\right) .
$$

In the rest frame of the solitary wave, for the unperturbed system, one has that

$$
T_{00}=h_{1}\left(1-\frac{1}{\kappa}\right)+h_{2},
$$

where

$$
\begin{aligned}
h_{1}= & R^{2}(x) \frac{d \theta}{d x}=\frac{\kappa \beta^{2}}{m+\omega \cosh (2 \kappa \beta x)} \\
& \times\left[\frac{(\kappa+1) \beta^{2}}{g^{2}(m+\omega \cosh (2 \kappa \beta x))}\right]^{1 / \kappa}, \\
h_{2}= & m \bar{\psi} \psi=m\left(A^{2}-B^{2}\right) \\
= & m\left[\frac{(\kappa+1) \beta^{2}}{g^{2}(m+\omega \cosh (2 \kappa \beta x))}\right]^{1 / \kappa} .
\end{aligned}
$$

Integrating in the rest frame, we get, for the rest-frame energy,

$$
E_{0}=H_{1}\left(1-\frac{1}{\kappa}\right)+H_{2}
$$

where

$$
\begin{aligned}
H_{1}= & \int d x h_{1}=\frac{\beta}{m+\omega}\left[\frac{(\kappa+1) \beta^{2}}{g^{2}(m+\omega)}\right]^{1 / \kappa} \\
& \times B\left(\frac{1}{2}, 1+\frac{1}{\kappa}\right){ }_{2} F_{1}\left(1+\frac{1}{\kappa}, \frac{1}{2}, \frac{3}{2}+\frac{1}{\kappa} ; \alpha^{2}\right), \\
H_{2}= & \int d x h_{2}=\frac{1}{\kappa \beta}\left[\frac{(\kappa+1) \beta^{2}}{g^{2}(m+\omega)}\right]^{1 / \kappa} \\
& \times B\left(\frac{1}{2}, \frac{1}{\kappa}\right){ }_{2} F_{1}\left(\frac{1}{\kappa}, \frac{1}{2}, \frac{1}{2}+\frac{1}{\kappa} ; \alpha^{2}\right) .
\end{aligned}
$$

Since in the rest frame for stationary solutions $T^{11}=T^{01}=$ 0 , the energy of the solitary wave in the moving frame is just

$$
E=E_{0} \cosh \eta=\gamma E_{0} ; \quad P=E_{0} \sinh \eta,
$$

so the norm $E^{2}-P^{2}=E_{0}^{2}=M_{0}^{2}$.

In particular, for $\kappa=1$ and $m=1$, we have that

$$
M_{0}=\frac{2}{g^{2} Q} \sinh ^{-1} \frac{g^{2} Q}{2} ; \quad Q=\frac{2 \sqrt{1-\omega^{2}}}{g^{2} \omega} .
$$

We also have

$$
\begin{aligned}
& H_{1}=-\frac{2\left[\sqrt{1-\omega^{2}}-2 \tanh ^{-1}\left(\sqrt{\frac{1-\omega}{1+\omega}}\right)\right]}{g^{2}}, \\
& H_{2}=\frac{4 \tanh ^{-1}\left(\sqrt{\frac{1-\omega}{1+\omega}}\right)}{g^{2}} .
\end{aligned}
$$

\section{STABILITY OF EXACT SOLUTIONS}

\section{A. Stability to changes in the frequency at fixed charge}

Bogolubsky [17] suggested that the stability could be ascertained by looking at variations of the wave function, keeping the charge fixed and seeing if the solution was a minimum (stable to that variation) or maximum (unstable to that variation) of the Hamiltonian as a function of the parameter $\omega$. This principle has been very useful in the past in determining the stability of scalar wave equations that are Hamiltonian dynamical systems. If the variation decreased the energy, it turned out that the solitary waves were unstable. Since in higher dimensions there are many degrees of freedom for perturbing the system, this criterion is a sufficient condition for instability. For the Dirac case, we have found from our numerical simulations that this criterion does not determine the critical $\omega$ except when $\kappa=1$ [18], the case originally studied by Bogolubsky [17]. Assuming we know the wave function at the value of $\omega$ corresponding to a fixed charge $Q$, if we change the parametric dependence on $\omega$, this also changes the charge. This can be corrected by assuming that the new wave function has a new normalization that corrects for this. That is, if we parametrize a rest-frame solitary-wave solution of the NLDE which has a charge $Q[\omega]$ by

$$
\psi_{s}(x, t)=\chi_{s}(x, \omega) e^{-i \omega t},
$$

then we choose our slightly changed wave function to be

$$
\begin{aligned}
\tilde{\psi}\left[x, t, \omega^{\prime}, \omega\right] & =\frac{\sqrt{Q[\omega]}}{\sqrt{Q\left[\omega^{\prime}\right]}} \chi_{s}\left(x, \omega^{\prime}\right) e^{-i \omega^{\prime} t} \\
& \equiv f\left(\omega^{\prime}, \omega\right) \chi_{s}\left(x, \omega^{\prime}\right) e^{-i \omega^{\prime} t} .
\end{aligned}
$$

The wave function $\tilde{\psi}\left[x, t, \omega^{\prime}, \omega\right]$ then has the same charge as $\psi[x, t, \omega]$. Inserting this wave function into the Hamiltonian, we get a new probe Hamiltonian $H_{p}$ depending on both $\omega^{\prime}, \omega$. As a function of $\omega^{\prime}$ this new Hamiltonian is stationary at the value $\omega^{\prime}=\omega$. The criterion Bogolubsky proposed [17] is that the solitary wave is stable (unstable) with respect to this variation in $\omega$ according to whether this new Hamiltonian has a minimum (maximum) at $\omega^{\prime}=\omega$. What we will find for $\kappa=1$ is that there is a critical value of $\omega$ (determined by the coupling $g$ and $Q$ ) below which the solitary wave is unstable, and this result is borne out by numerical simulations 
which we will present below. However, we will present in another paper numerical simulations at arbitrary $\kappa$ which suggest that this method provides only a sufficient condition for instability [18]. The probe Hamiltonian has the form

$$
\begin{aligned}
H_{p}\left[\omega^{\prime}, \omega\right]= & H_{1}\left[\omega^{\prime}\right]\left[f\left(\omega^{\prime}, \omega\right)^{2}-\frac{1}{\kappa} f\left(\omega^{\prime}, \omega\right)^{2(\kappa+1)}\right] \\
& +H_{2}\left[\omega^{\prime}\right] f\left(\omega^{\prime}, \omega\right)^{2} .
\end{aligned}
$$

For $\kappa=1$ we have that $f\left(\omega^{\prime}, \omega\right)^{2}=\frac{\beta[\omega] \omega^{\prime}}{\beta\left[\omega^{\prime}\right] \omega}$, where $\beta[\omega]=\sqrt{1-\omega^{2}}$. We then find that the first derivative of $H_{p}$ with respect to $\omega^{\prime}$ evaluated at $\omega^{\prime}=\omega$ is indeed zero. The second derivative evaluated at $\omega^{\prime}=\omega$ leads to the following expression:

$$
\left.\frac{\partial^{2} H_{p}}{\partial \omega^{\prime 2}}\right|_{\omega=\omega^{\prime}}=-\frac{2\left[\sqrt{1-\omega^{2}}\left(\omega^{2}-3\right)+4 \tanh ^{-1}\left(\sqrt{\frac{1-\omega}{1+\omega}}\right)\right]}{g^{2} \omega^{2}\left(\omega^{2}-1\right)^{2}} .
$$

This function is zero at $\omega_{c}=0.697586$ and the second derivative is negative below this value of $\omega$ showing an instability. In our numerical simulations of the unforced NLDE [18], we find that, below this value, the solitary waves are metastable, with the time for the instability to set in increasing exponentially as a function of $\omega$ for $\omega<\omega_{c}$.

\section{NLDE IN EXTERNAL ELECTROMAGNETIC FIELDS}

We add electromagnetic interactions through the gauge covariant derivative

$$
i \partial_{\mu} \Psi \rightarrow\left(i \partial_{\mu}-e A_{\mu}\right) \Psi
$$

and then, under the combined transformations

$\Psi \rightarrow e^{i \Lambda(x)} \Psi ; \quad A_{\mu} \rightarrow A_{\mu}-\frac{1}{e} \partial_{\mu} \Lambda ; \quad \bar{\Psi} \rightarrow \bar{\Psi} e^{-i \Lambda(x)}$,

the Lagrangian is invariant. Again, the conserved current is given by Eq. (2.34). The gauge-invariant Lagrangian for the external field problem is

$$
\begin{aligned}
L= & \left(\frac{i}{2}\right)\left[\bar{\Psi} \gamma^{\mu} \partial_{\mu} \Psi-\partial_{\mu} \bar{\Psi} \gamma^{\mu} \Psi\right]-m \bar{\Psi} \Psi \\
& +\frac{g^{2}}{\kappa+1}(\bar{\Psi} \Psi)^{\kappa+1}-e \bar{\Psi} \gamma^{\mu} A_{\mu} \Psi .
\end{aligned}
$$

One again finds that energy-momentum is conserved, with $E$ and $P$ given by Eq. (2.38). Because of Lorentz invariance, it is sufficient to calculate the energy-momentum tensor in the comoving frame $v=0$. The energy-momentum tensor in an arbitrary frame is then given by Eq. (2.39). Using the freedom of gauge invariance, one can choose the axial gauge $A_{1}=0$, $e A_{0}=V(x)$. In the axial gauge the Dirac equation becomes

$$
i \gamma^{\mu} \partial_{\mu} \Psi-m \Psi+g^{2}(\bar{\Psi} \Psi)^{\kappa} \Psi-\gamma^{0} V(x) \Psi=0 .
$$

Going into the rest frame and choosing for $\psi_{0}$ the representation of Eq. (2.4), we find that the Dirac equation becomes

$$
\begin{aligned}
& \partial_{x} A+(m+\omega) B-g^{2}\left[A^{2}-B^{2}\right]^{\kappa} B-V(x) B=0, \\
& \partial_{x} B+(m-\omega) A-g^{2}\left[A^{2}-B^{2}\right]^{\kappa} A+V(x) A=0 .
\end{aligned}
$$

As in the case of the standard NLDE equation, we can again look for static solutions of the form $\psi_{0}$, where $A=R \cos \theta$ and $B=R \sin \theta$. The conservation of $T^{11}$ for static solutions that vanish at infinity yields the equation

$$
T^{11}=\omega \psi^{\dagger} \psi-m \bar{\psi} \psi+\mathcal{L}_{I}=0,
$$

where

$$
\mathcal{L}_{I}=\frac{g^{2}}{\kappa+1}(\bar{\psi} \psi)^{\kappa+1}-V(x) \psi^{\dagger} \psi .
$$

Multiplying the Dirac equation [Eq. (4.4)] on the left by $\bar{\Psi}$ and using Eq. (2.3), we obtain

$$
\omega \psi^{\dagger} \psi+i \bar{\psi} \gamma^{1} \partial_{x} \psi-m \bar{\psi} \psi+g^{2}(\bar{\psi} \psi)^{\kappa+1}-V(x) \psi^{\dagger} \psi=0 .
$$

The energy density is given by

$$
\begin{aligned}
h= & \frac{i}{2}\left[\bar{\Psi} \gamma^{1} \partial_{x} \Psi-\partial_{x} \bar{\Psi} \gamma^{1} \Psi\right]+m \bar{\Psi} \Psi \\
& -\frac{g^{2}}{\kappa+1}(\bar{\Psi} \Psi)^{\kappa+1}+V(x) \Psi^{\dagger} \Psi \\
\equiv & h_{1}+h_{2}-h_{3}+h_{4} .
\end{aligned}
$$

From Eq. (4.6), (4.7), and Eq. (4.8) we again find that

$$
h_{3}=\frac{1}{\kappa} h_{1} \text {. }
$$

Multiplying the Dirac equation [Eq. (4.4)] on the left by $\bar{\Psi}$ and using Eq.(2.3), eliminating the self-interaction term using Eq. (4.6), we obtain

$$
i \bar{\psi} \gamma^{1} \partial_{x} \psi+\kappa\left[m \bar{\psi} \psi+V(x) \psi^{\dagger} \psi-\omega \psi^{\dagger} \psi\right]=0 .
$$

Realizing that the equations for the standard NLDE are modified by replacing $\omega$ by $\omega-V(x)$, we obtain, for $\theta(x)$,

$$
\frac{d \theta}{d x}=-\kappa \omega+\kappa m \cos (2 \theta)+\kappa V(x)
$$

and for $R(x)$ we obtain

$$
R^{2}=\left[\frac{(\kappa+1)(m \cos 2 \theta-\omega+V(x))}{g^{2}(\cos 2 \theta)^{\kappa+1}}\right]^{1 / \kappa} .
$$

Notice, if we now choose $V(x)=\mu \cos 2 \theta$, we arrive at the solutions found earlier with $m \rightarrow m+\mu$. Note that for a bound state without the external potential we needed $\omega<m$. So now we need $\omega<m+\mu$ for $\beta_{k}$ to be real. The potential is then

$$
\begin{aligned}
V_{1}(x) & =\mu \frac{(m+\mu)+\omega \cosh 2 \beta_{\kappa} x}{\omega+(m+\mu) \cosh 2 \beta_{\kappa} x} ; \\
\beta_{\kappa} & =\kappa \sqrt{(m+\mu)^{2}-\omega^{2}} .
\end{aligned}
$$

The eigenvalue $\omega$ is set by fixing the charge $Q$ of the solitary wave. We can choose a negative $\mu$ satisfying $\omega<m+\mu$ so the potential at small $x$ looks much like a harmonic trap. Namely, choosing $\kappa=1, \mu=-1 / 4, m=1, \omega=1 / 2$, then

$$
V_{1}(x)=-\frac{2 \cosh \left(\frac{\sqrt{5} x}{2}\right)+3}{12 \cosh \left(\frac{\sqrt{5} x}{2}\right)+8} .
$$

This is plotted in Fig. 1. The charge density for the solitary wave corresponding to this external potential is plotted in Fig. 2. 


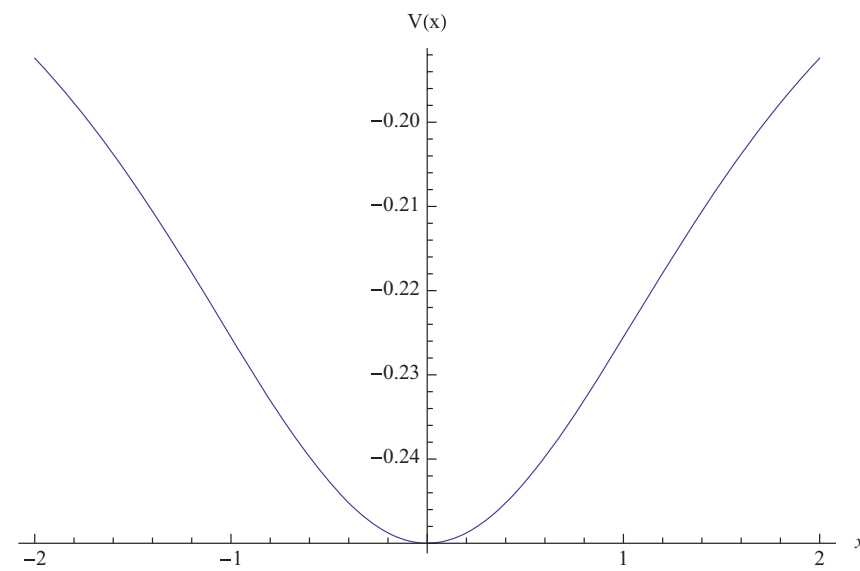

FIG. 1. (Color online) Potential $V_{1}(x)$ corresponding to Eq. (4.14) with $\mu=-1 / 4, \omega=1 / 2$.

At small $x, V(x)$ has the expansion

$$
V_{1}(x) \simeq-\frac{1}{4}+\frac{x^{2}}{32}-\frac{13 x^{4}}{1536}+O\left(x^{6}\right)
$$

Another solution is found if we let

$$
V_{2}(x)=\mu \sin (2 \theta(x)) .
$$

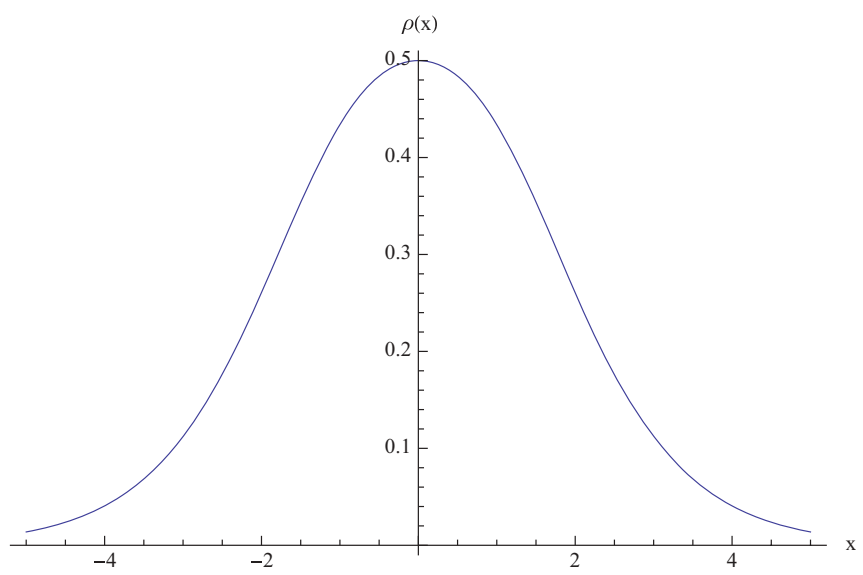

FIG. 2. (Color online) Charge density $R^{2}(x)$ corresponding to the potential $V_{1}(x)$ given by Eq. (4.14) with $\mu=-1 / 4$, $\omega=1 / 2$.

The solution to the differential equation,

$$
\theta^{\prime}(x)=-\kappa \omega+\kappa m \cos (2 \theta(x))+\kappa \mu \sin (2 \theta(x)),
$$

then is found to be

$$
\theta(x)=\tan ^{-1}\left\{\frac{\mu+\sqrt{\mu^{2}+m^{2}-\omega^{2}} \tanh \left[\kappa\left(x-x_{0}\right) \sqrt{\mu^{2}+m^{2}-\omega^{2}}\right]}{m+\omega}\right\} .
$$

Letting $x_{0}=0$, we have that

$$
\tan \theta=\frac{\mu+\sqrt{\mu^{2}+m^{2}-\omega^{2}} \tanh \left(\kappa x \sqrt{\mu^{2}+m^{2}-\omega^{2}}\right)}{m+\omega} .
$$

The potential is, thus, given by

$$
V_{2}(x)=\mu \sin 2 \theta=2 \mu \frac{\tan \theta}{1+\tan ^{2} \theta}=\frac{2 \mu\left[\mu+\sqrt{\mu^{2}+m^{2}-\omega^{2}} \tanh \left(\kappa x \sqrt{\mu^{2}+m^{2}-\omega^{2}}\right)\right]}{(m+\omega)\left\{\frac{\left[\mu+\sqrt{\mu^{2}+m^{2}-\omega^{2}} \tanh \left(\kappa x \sqrt{\mu^{2}+m^{2}-\omega^{2}}\right)\right]^{2}}{(m+\omega)^{2}}+1\right\}} .
$$

For the values $\kappa=1, \mu=-1 / 4, m=1, \omega=1 / 2$, we find that $V_{2}(x)$ has a kinklike form shown in Fig. 3 . The charge density $R^{2}$ corresponding to these values of the parameters in $V_{2}(x)$ is shown in Fig. 4.

For small $x$ for these parameters

$$
V_{2}(x) \simeq \frac{3}{37}-\frac{1365 x}{5476}-\frac{54249 x^{2}}{810448}+O\left(x^{3}\right)
$$

and if instead we choose $\mu=1 / 4$ and leave the other parameters the same, we get the opposite type of kink shown in Fig. 5 . Now for small $x$ we have

$$
V_{2}(x) \simeq \frac{3}{37}+\frac{1365 x}{5476}-\frac{54249 x^{2}}{810448}+O\left(x^{3}\right) .
$$

We can also solve for the more general case

$$
V_{3}(x)=\mu_{1} \cos (2 \theta(x))+\mu_{2} \sin (2 \theta(x)),
$$

and then the solution to the differential equation is

$$
\theta^{\prime}(x)=-\kappa \omega+\kappa\left(m+\mu_{1}\right) \cos (2 \theta(x))+\kappa \mu_{2} \sin (2 \theta(x)),
$$

so we just need to change $m \rightarrow m+\mu_{1}$ and $\mu \rightarrow \mu_{2}$ to obtain

$$
\tan \theta=\frac{\mu_{2}+\sqrt{\mu_{2}^{2}+\left(m+\mu_{1}\right)^{2}-\omega^{2}} \tanh \left[\kappa x \sqrt{\mu_{2}^{2}+\left(m+\mu_{1}\right)^{2}-\omega^{2}}\right]}{m+\mu_{1}+\omega} .
$$


The potential is then given by

$$
V_{3}(x)=\mu_{1} \cos 2 \theta+\mu_{2} \sin 2 \theta=\mu_{1} \frac{1-\tan ^{2} \theta}{1+\tan ^{2} \theta}+2 \mu_{2} \frac{\tan \theta}{1+\tan ^{2} \theta}=\frac{N(x)}{D(x)},
$$

where

$$
\begin{aligned}
N(x) & =\frac{2 \mu_{2}\left\{\mu_{2}+\sqrt{\mu_{2}^{2}+\left(\mu_{1}+m\right)^{2}-\omega^{2}} \tanh \left[\kappa x \sqrt{\mu_{2}^{2}+\left(\mu_{1}+m\right)^{2}-\omega^{2}}\right]\right\}}{\mu_{1}+m+\omega} \\
& +\mu_{1}\left(1-\frac{\left\{\mu_{2}+\sqrt{\mu_{2}^{2}+\left(\mu_{1}+m\right)^{2}-\omega^{2}} \tanh \left[\kappa x \sqrt{\mu_{2}^{2}+\left(\mu_{1}+m\right)^{2}-\omega^{2}}\right]\right\}^{2}}{\left(\mu_{1}+m+\omega\right)^{2}}\right)
\end{aligned}
$$

and

$$
D(x)=\frac{\left\{\mu_{2}+\sqrt{\mu_{2}^{2}+\left(\mu_{1}+m\right)^{2}-\omega^{2}} \tanh \left[\kappa x \sqrt{\mu_{2}^{2}+\left(\mu_{1}+m\right)^{2}-\omega^{2}}\right]\right\}^{2}}{\left(\mu_{1}+m+\omega\right)^{2}}+1 .
$$

For $\omega=1 / 2, \mu_{1}=\mu_{2}=-1 / 4, \kappa=1, m=1, V_{3}(x)$ has the form of Fig. 6 . The charge density corresponding to this potential is given in Fig. 7. for $t$,

One can also consider the potential $V_{4}=\mu \tan ^{2} \theta$. In that case, on substituting $t=\tan \theta$, we obtain the differential equation

$$
\frac{d t}{d x}=\kappa\left[t^{2}(\mu-m-\omega)+m+\mu t^{4}-\omega\right]
$$

Thus, we obtain

$$
\begin{gathered}
\kappa x=\frac{\sqrt{2 \mu} \tan ^{-1}\left[\frac{\sqrt{2 \mu} t}{\sqrt{\mu-\sqrt{(\mu+\omega)^{2}+m^{2}+2 m(\omega-3 \mu)}-m-\omega}}\right]}{\sqrt{(\mu+\omega)^{2}+m^{2}+2 m(\omega-3 \mu)} \sqrt{\mu-\sqrt{(\mu+\omega)^{2}+m^{2}+2 m(\omega-3 \mu)}-m-\omega}} \\
-\frac{\sqrt{2 \mu} \tan ^{-1}\left[\frac{\sqrt{2 \mu} t}{\sqrt{\mu+\sqrt{(\mu+\omega)^{2}+m^{2}+2 m(\omega-3 \mu)}-m-\omega}}\right]}{\sqrt{(\mu+\omega)^{2}+m^{2}+2 m(\omega-3 \mu)} \sqrt{\mu+\sqrt{(\mu+\omega)^{2}+m^{2}+2 m(\omega-3 \mu)}-m-\omega}} .
\end{gathered}
$$

\section{A. " $\omega$ " stability of exact solutions with $\kappa=1$ in the presence of an external field}

Here we follow the method of Bogolubsky [17] and consider stability to changes in the frequency $\omega$, keeping the charge $Q$ fixed. That is, if we parametrize a rest-frame

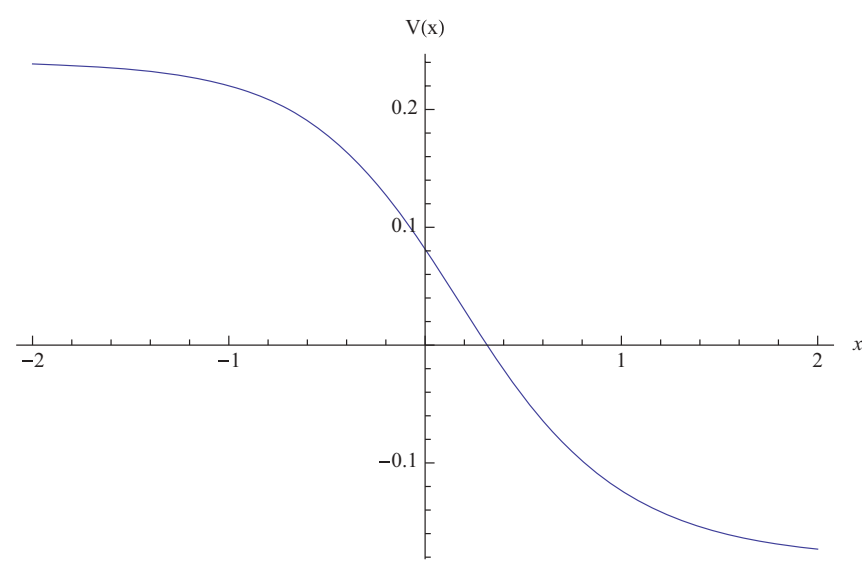

FIG. 3. (Color online) Potential $V_{2}(x)$ corresponding to the exact solution Eq. (4.21) with $\omega=1 / 2, \mu=-1 / 4$. solitary-wave solution by

$$
\psi_{s}(x, t)=\chi_{s}(x, \omega) e^{-i \omega t},
$$

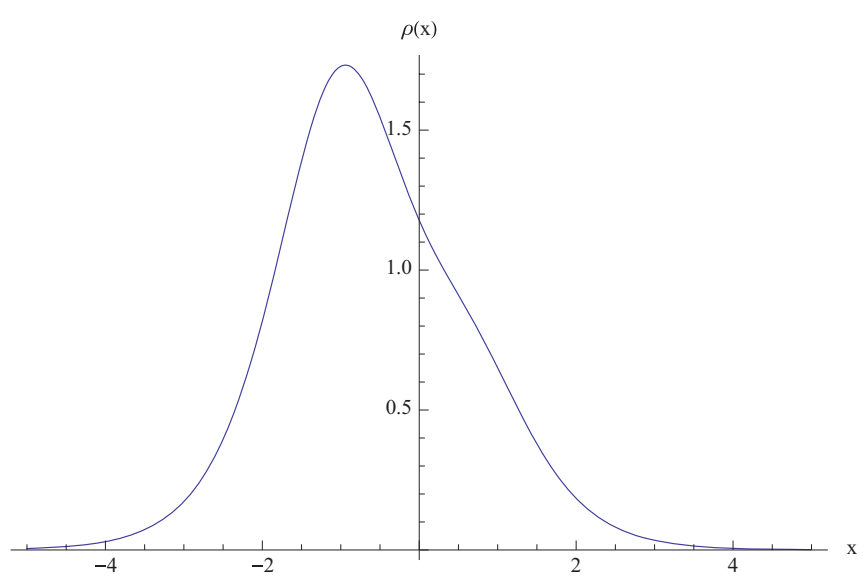

FIG. 4. (Color online) The charge density for the solitary wave in the potential $V_{2}(x)$ given Eq. (4.21) with $\omega=1 / 2, \mu=-1 / 4$. 


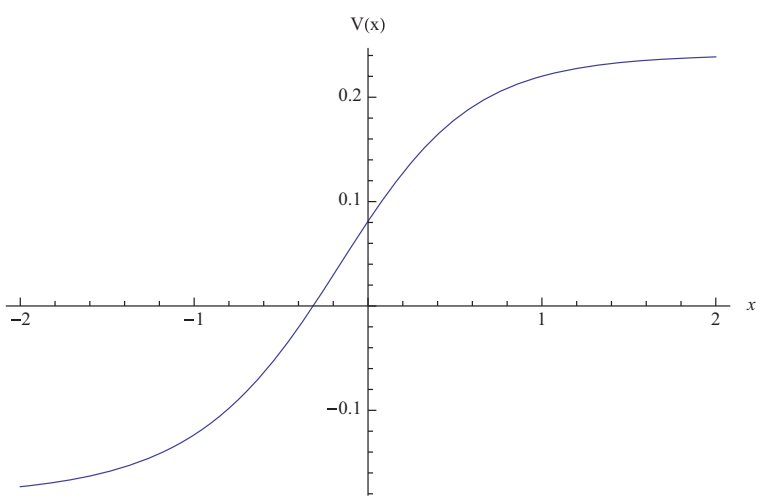

FIG. 5. (Color online) Potential $V_{2}(x)$ corresponding to the exact solution Eq. (4.21) with $\mu=1 / 4$.

then we choose our perturbed wave function to be

$$
\begin{aligned}
\tilde{\psi}\left[x, t, \omega^{\prime}, \omega\right] & =\frac{\sqrt{Q[\omega]}}{\sqrt{Q\left[\omega^{\prime}\right]}} \chi_{s}\left(x, \omega^{\prime}\right) e^{-i \omega^{\prime} t} \\
& \equiv f\left(\omega^{\prime}, \omega\right) \chi_{s}\left(x, \omega^{\prime}\right) e^{-i \omega^{\prime} t} .
\end{aligned}
$$

Again inserting this wave function into the Hamiltonian, we get a new probe Hamiltonian $H_{p}$ depending on both $\omega^{\prime}, \omega$. We will consider the most general solution we have found where the potential has the form of $V_{3}(x)$ given by Eq. (4.24). The energy density is given by the four terms in Eq. (4.9), $\left(h_{1}+h_{2}-h_{3}+h_{4}\right)$, where

$$
\begin{aligned}
& h_{1}=R^{2} \frac{d \theta}{d x} ; \quad h_{2}=m R^{2} \cos 2 \theta, \\
& h_{3}=\frac{g}{2}\left(R^{2} \cos 2 \theta\right)^{2} ; \quad h_{4}=R^{2}\left(\mu_{1} \cos 2 \theta+\mu_{2} \sin 2 \theta\right) .
\end{aligned}
$$

We also have that $h_{3}=h_{1}$. Following our previous discussion, the probe Hamiltonian has the form

$$
\begin{aligned}
H_{p}\left[\omega^{\prime}, \omega\right]= & H_{1}\left[\omega^{\prime}\right]\left[f\left(\omega^{\prime}, \omega\right)^{2}-f\left(\omega^{\prime}, \omega\right)^{4}\right] \\
& +\left(H_{2}+H_{4}\right)\left[\omega^{\prime}\right] f\left(\omega^{\prime}, \omega\right)^{2} .
\end{aligned}
$$

For our choice of potential, we have the following relationship:

$$
\frac{d \theta}{d x}=\left(m+\mu_{1}\right) \cos 2 \theta+\mu_{2} \sin 2 \theta-\omega .
$$

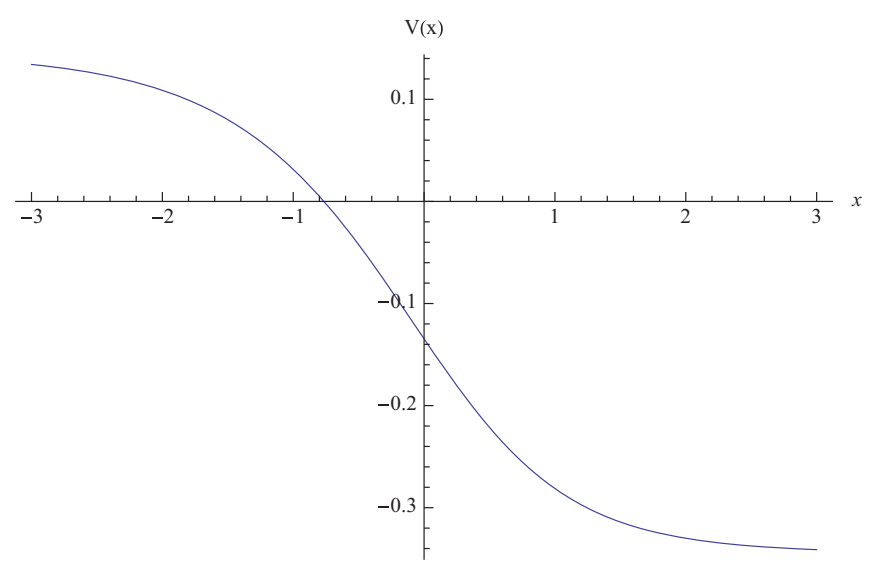

FIG. 6. (Color online) Potential $V_{3}(x)$ corresponding to Eq. (4.27) with $\omega=1 / 2, \mu_{1}=\mu_{2}=-1 / 4$.

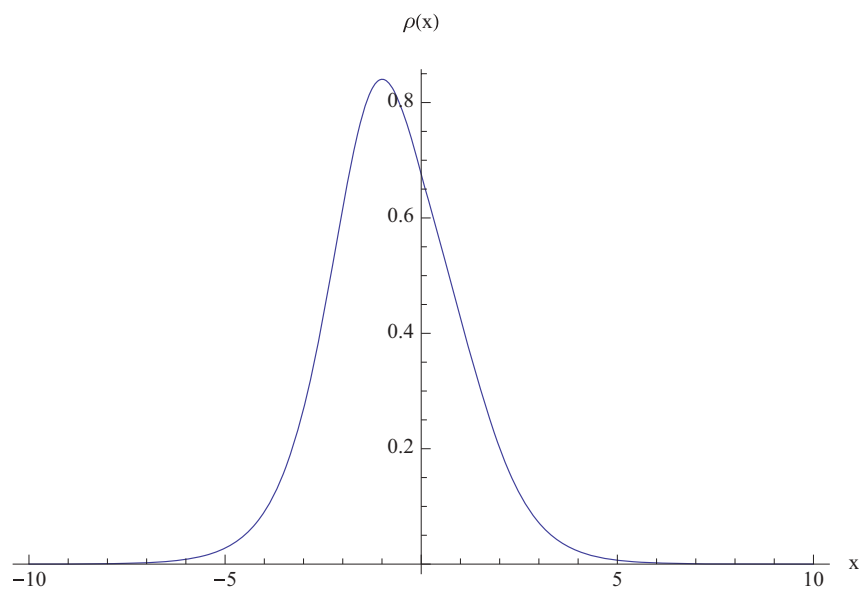

FIG. 7. (Color online) Charge density for the solitary wave in the potential $V_{3}(x)$ corresponding to Eq. (4.27) with $\omega=1 / 2, \mu_{1}=$ $\mu_{2}=-1 / 4$.

Solving this equation, we obtain

$$
\tan \theta \equiv T=D+E \tanh B x,
$$

where

$$
\begin{aligned}
& B=\sqrt{\left(m+\mu_{1}\right)^{2}+\mu_{2}^{2}-\omega^{2}} ; \quad E=\frac{B}{d_{1}} ; \\
& D=\frac{\mu_{2}}{d_{1}} ; \quad d_{1}=m+\mu_{1}+\omega .
\end{aligned}
$$

We also find

$$
\begin{aligned}
R^{2} & =\frac{2}{g^{2}}\left[\frac{\left(m+\mu_{1}\right) \cos 2 \theta+\mu_{2} \sin 2 \theta-\omega}{\cos ^{2} 2 \theta}\right] \\
& =\frac{2 B^{2}}{d_{1} g^{2}} \operatorname{sech}^{2} B x \frac{\left[1+(D+E \tanh B x)^{2}\right]}{\left[1-(D+E \tanh B x)^{2}\right]^{2}} .
\end{aligned}
$$

The charge is given by

$$
Q[\omega]=\int_{-\infty}^{\infty} d x R^{2}=\frac{2 \omega \sqrt{\left(m+\mu_{1}\right)^{2}+\mu_{2}^{2}-\omega^{2}}}{g^{2}\left(\omega^{2}-\mu_{2}^{2}\right)}
$$

Also,

$$
H_{1}=-\omega Q+H_{2}+H_{4} ; \quad H_{2}+H_{4}=\left(m+\mu_{1}\right) T_{1}+\mu_{2} T_{2},
$$

where

$$
\begin{aligned}
T_{1} & =\int d x R^{2} \cos 2 \theta=2\left[\tanh ^{-1}(E-D)+\tanh ^{-1}(E+D)\right] \\
& =\frac{2}{g^{2}} \tanh ^{-1}\left(\frac{\sqrt{\left(\mu_{1}+m\right)^{2}+\mu_{2}^{2}-\omega}}{\mu_{1}+m}\right), \\
T_{2} & =\int d x R^{2} \sin 2 \theta \\
& =\frac{2 \mu_{2}\left(m+\mu_{1}\right)}{g^{2}\left(\omega^{2}-\mu_{2}^{2}\right)}-\frac{2 \mu_{2}}{g^{2}} \frac{\tanh ^{-1}\left(\frac{\sqrt{\left(\mu_{1}+m\right)^{2}+\mu_{2}^{2}-\omega^{2}}}{m+\mu_{1}}\right)}{\sqrt{\left(\mu_{1}+m\right)^{2}+\left(\mu_{2}^{2}-\omega^{2}\right)}} .
\end{aligned}
$$


Thus,

$$
\begin{aligned}
H_{p}\left[\omega^{\prime}\right]= & -\omega^{\prime} Q\left[\omega^{\prime}\right] f\left(\omega^{\prime}, \omega\right)^{2}\left[1-f\left(\omega^{\prime}, \omega\right)^{2}\right] \\
& +f\left(\omega^{\prime}, \omega\right)^{2}\left(H_{2}\left[\omega^{\prime}\right]+H_{4}\left[\omega^{\prime}\right]\right)\left[2-f\left(\omega^{\prime}, \omega\right)^{2}\right] .
\end{aligned}
$$

If we take the first derivative of this probe Hamiltonian with respect to $\omega^{\prime}$ and set $\omega^{\prime}=\omega$, we find that this is zero only when $\mu_{2}=0$. Thus, the probe Hamiltonian is stationary to $\omega$ variations only when $\mu_{2}=0$. However, we can use this method for the external potential $V_{1}(x)=\mu_{1} \cos 2 \theta$ [see Eq. (4.14)]. In that case, the first derivative is automatically zero when $\omega^{\prime}=\omega$. The second derivative changes sign at a particular value of $\omega=\omega^{\star}$, which, for $m=1$, is the solution to the equation

$$
\begin{gathered}
\frac{2\left(\mu_{1}+1\right)^{3} \operatorname{coth}^{-1}\left[\frac{\mu_{1}+1}{\sqrt{\mu_{1}\left(\mu_{1}+2\right)-\omega^{2}+1}}\right]}{\sqrt{\mu_{1}^{2}+2 \mu_{1}-\omega^{2}+1}} \\
-3 \mu_{1}^{2}-6 \mu_{1}+\omega^{2}-3=0 .
\end{gathered}
$$

Now we have that for there to be an allowed real solution $\omega<m+\mu_{1}$. So if $\mu_{1}$ is positive, $\omega^{\star}$ is shifted upward compared to $\omega_{c}$ and so is the region in $\omega$ space where the solutions are real. If $\mu_{1}$ is negative $\omega^{\star}$ is shifted downward, but also the allowed regime of $\omega$ is decreased. The net result is that the possible regime of stability is approximately $30 \%$ of the allowed region for real solutions independent of the value of $\mu_{1}$. Preliminary simulations show that some of the exact solutions with the potential (4.14) are metastable. For the well potential, $\mu_{1}<0$, the solitary wave is metastable, probably due to the fact that the initial solitary wave extends beyond the inflection points of the potential well. Details of these simulations will be discussed in a future paper.

\section{VARIATIONAL ANSATZ FOR THE NLDE IN EXTERNAL FIELDS}

The gauge-invariant Lagrangian for the external field problem is given by Eq. (4.3). Using the freedom of gauge invariance, one can choose the axial gauge $A_{1}=0, e A_{0}=$ $V(x)$. Our ansatz for the trial variational wave function is to assume that, because of the smallness of the perturbation, the main modification to our exact solutions to the NLDE equation without an external field is that the parameters describing the position, momentum, boost, and phase become time dependent. That is, we replace

$$
\begin{aligned}
v t & \rightarrow q(t) ; \quad \eta \rightarrow \eta(t) ; \quad \gamma \omega v \rightarrow p(t) ; \\
\omega t^{\prime} & =\gamma \omega(t-v x) \rightarrow \phi(t)-p(t)[x-q(t)],
\end{aligned}
$$

where $\phi(t)=\omega \gamma t-p(t) q(t)$. by

Thus, our trial wave function in component form is given

$$
\begin{aligned}
& \Psi_{1}(x, t)=\left[\cosh \frac{\eta}{2} A\left(x^{\prime}\right)+i \sinh \frac{\eta}{2} B\left(x^{\prime}\right)\right] e^{-i \phi+i p(x-q)}, \\
& \Psi_{2}(x, t)=\left[\sinh \frac{\eta}{2} A\left(x^{\prime}\right)+i \cosh \frac{\eta}{2} B\left(x^{\prime}\right)\right] e^{-i \phi+i p(x-q)},
\end{aligned}
$$

where $x^{\prime}=\cosh \eta(t)[x-q(t)]$. Using this trial wave function we can determine the effective Lagrangian for the variational parameters. Writing the Lagrangian density as

$$
\mathcal{L}=\mathcal{L}_{1}+\mathcal{L}_{2}+\mathcal{L}_{3},
$$

where

$$
\begin{aligned}
& \mathcal{L}_{1}=\frac{i}{2}\left(\bar{\Psi} \gamma^{\mu} \partial_{\mu} \Psi-\partial_{\mu} \bar{\Psi} \gamma^{\mu} \Psi\right), \\
& \mathcal{L}_{2}=-m \bar{\Psi} \Psi+\frac{g^{2}}{\kappa+1}(\bar{\Psi} \Psi)^{\kappa+1}, \\
& \mathcal{L}_{3}=-e A_{0} \bar{\Psi} \gamma^{0} \Psi \equiv-V(x) \Psi^{\dagger} \Psi .
\end{aligned}
$$

Integrating over $x$ and changing integration variables to $z=$ $(x-q) \cosh \eta$, one obtains

$$
\begin{aligned}
L_{1} & =\int d x \mathcal{L}_{1} \\
& =Q(p \dot{q}+\dot{\phi}-p \tanh \eta)-I_{0}(\cosh \eta-\dot{q} \sinh \eta),
\end{aligned}
$$

where

$$
Q=\int d z\left[A^{2}(z)+B^{2}(z)\right]
$$

is as given by Eq. (2.36). Note that

$$
I_{0}=\int d z\left(B^{\prime} A-A^{\prime} B\right)=H_{1},
$$

where $H_{1}$ is the rest-frame kinetic energy and is given by Eq. (2.44). Here $B^{\prime}\left(x^{\prime}\right)=\frac{d B\left(x^{\prime}\right)}{d x^{\prime}}$, and

$$
L_{2}=\int \mathcal{L}_{2} d x=-\frac{m}{\cosh \eta} I_{1}+\frac{g^{2}}{(\kappa+1) \cosh \eta} I_{2},
$$

where

$I_{1}=\int d z\left[A^{2}(z)-B^{2}(z)\right] ; \quad I_{2}=\int d z\left[A^{2}(z)-B^{2}(z)\right]^{(\kappa+1)}$,

and

$$
L_{3}=-\int d z \rho(z) V\left[\frac{z}{\cosh \eta}+q(t)\right]=-U[\eta(t), q(t)] .
$$

Putting these terms together, we obtain

$$
\begin{aligned}
L= & Q(p \dot{q}+\dot{\phi}-p \tanh \eta)-I_{0}(\cosh \eta-\dot{q} \sinh \eta) \\
& -\frac{m}{\cosh \eta} I_{1}+\frac{g^{2}}{(\kappa+1) \cosh \eta} I_{2}-U[\eta(t), q(t)] .
\end{aligned}
$$

We now get the following Lagrange's equations:

$$
\frac{d}{d t} \frac{\delta L}{\delta \dot{\phi}}=0 \rightarrow \frac{d Q}{d t}=0 \rightarrow Q=\text { const, }
$$

i.e., the charge is canonically conjugated to the phase $\phi$. The canonical solitary-wave momentum, which is conjugated to the solitary-wave position, is

$$
\begin{aligned}
P_{q} & =\frac{\delta L}{\delta \dot{q}}=Q p+I_{0} \sinh \eta, \\
\frac{d P_{q}}{d t} & =Q \dot{p}+I_{0} \cosh \eta \dot{\eta}=\frac{\delta L}{\delta q}=-\frac{\partial U}{\partial q},
\end{aligned}
$$


from

$$
\frac{\delta L}{\delta p}=0 \rightarrow \dot{q}=\tanh \eta,
$$

which implies $\sinh \eta=\gamma \dot{q}$ and $\cosh \eta=\gamma=\left(1-\dot{q}^{2}\right)^{-1}$. Also,

$$
\begin{aligned}
\frac{\delta L}{\delta \eta}= & 0 \rightarrow-Q p \operatorname{sech}^{2} \eta-I_{0}(\sinh \eta-\dot{q} \cosh \eta) \\
& +\tanh \eta \operatorname{sech} \eta\left[m I_{1}-\frac{g^{2}}{(\kappa+1)} I_{2}\right]-\frac{\partial U}{\partial \eta} \\
= & 0 .
\end{aligned}
$$

Changing variables to $\dot{q}=\tanh \eta$ and using $\frac{d \dot{q}}{d \eta}=\operatorname{sech}^{2} \eta$, we obtain

$$
Q p(t)=\gamma \dot{q}\left[m I_{1}-\frac{g^{2}}{(\kappa+1)} I_{2}\right]-\frac{\partial U}{\partial \dot{q}}
$$

and

$$
Q \dot{p}+I_{0} \gamma \dot{q}=\frac{\delta L}{\delta q}=-\frac{\partial U}{\partial q} .
$$

From Eq. (5.15) we also have

$$
Q \dot{p}=\frac{d(\gamma \dot{q})}{d t}\left[m I_{1}-\frac{g^{2}}{(\kappa+1)} I_{2}\right]-\frac{d}{d t} \frac{\partial U}{\partial \dot{q}} .
$$

Combining Eqs. (5.16) and (5.17), we obtain an equation for the generalized force $F_{\text {eff }}$

$$
\mu \frac{d(\gamma \dot{q})}{d t}=F_{\mathrm{eff}}[q, \dot{q}]
$$

where

$$
\mu=m I_{1}-\frac{g^{2}}{(\kappa+1)} I_{2}+I_{0} ; \quad F_{\mathrm{eff}}[q, \dot{q}]=\frac{d}{d t} \frac{\partial U}{\partial \dot{q}}-\frac{\partial U}{\partial q} .
$$

Now for the NLDE without the presence of external forces, the solitary wave in the frame with $v=0$ obeys Eq. (2.9)

$$
\omega \psi^{\dagger} \psi-m \bar{\psi} \psi+\frac{g^{2}}{\kappa+1}(\bar{\psi} \psi)^{\kappa+1}=0 .
$$

For our problem this converts into

$$
\omega\left(A^{2}+B^{2}\right)-m\left(A^{2}-B^{2}\right)+\frac{g^{2}}{(\kappa+1)}\left(A^{2}-B^{2}\right)^{(\kappa+1)}=0 .
$$

Integrating this relationship, we obtain

$$
m I_{1}-\frac{g^{2}}{(\kappa+1)} I_{2}=\omega Q,
$$

and, thus, we can write Eq. (5.18) as

$$
\frac{d(M \dot{q})}{d t}=F_{\text {eff }}[q, \dot{q}]
$$

where

$$
M=\left(Q \omega+I_{0}\right) \gamma=M_{0} \gamma .
$$

Here $F_{\text {eff }}$ is given by Eq. (5.19), where

$$
U(\dot{q}, q)=\int_{-\infty}^{\infty} d z V\left(q+\frac{z}{\gamma}\right)\left[A^{2}(z)+B^{2}(z)\right]
$$

and $\gamma=1 / \sqrt{1-\dot{q}^{2}}$. We can rewrite the relativistic force equation as

$$
\gamma^{3} M_{0} \ddot{q}=F_{\mathrm{eff}}[q, \dot{q}] .
$$

Using the rest frame identities of the appendix, we have that

$$
M_{0}=I_{0}+\omega Q \text {. }
$$

Here $I_{0}$ is given by Eq. (5.7), and $Q$ is given by Eq. (2.36).

It is useful to rewrite the equation for the canonical momentum $P_{q}$ using the definition of $M_{0}$ and Eq. (5.15) as follows:

$$
P_{q}=Q p+I_{0} \gamma \dot{q}=M_{0} \gamma \dot{q}-\frac{\partial U[q, \dot{q}]}{\partial \dot{q}} .
$$

\section{A. Energy-momentum tensor}

The fact that the external potential is explicitly independent of time means that the energy of the solitary wave is independent of time. The energy density is given by

$$
T^{00}=\frac{i}{2}\left(\bar{\psi} \gamma^{0} \partial_{t} \psi-\partial_{t} \bar{\psi} \gamma^{0} \psi\right)-\mathcal{L}
$$

Straightforward integration leads to

$$
\begin{aligned}
E & =\int d x T^{00} \\
& =Q p \dot{q}+\gamma I_{0}+\frac{m}{\gamma} I_{1}-\frac{g^{2}}{(\kappa+1) \gamma} I_{2}+U[q, \dot{q}] .
\end{aligned}
$$

Using the identities Eq. (5.22) and Eq. (5.24), we can rewrite this as

$$
E=M_{0} \gamma+Q p \dot{q}-\gamma \omega Q \dot{q}^{2}+U[q, \dot{q}] .
$$

From (5.15) and (5.22) we have that

$$
Q p=\gamma \dot{q} \omega Q-\frac{\partial U}{\partial \dot{q}},
$$

and, thus, we can write the energy of the solitary wave in the following convenient form:

$$
E=M_{0} \gamma+U[q, \dot{q}]-\dot{q} \frac{\partial U}{\partial \dot{q}} .
$$

The conservation of energy will be important to test our numerical integration schemes in Sec. VII.

For time-independent external forces the total momentum of the solitary wave is not conserved but changes depending on the external force. We have that

$$
P=\int T^{01} d x=-\frac{i}{2} \int d x\left(\psi^{\dagger} \partial_{x} \psi-\partial_{x} \psi^{\dagger} \psi\right) .
$$

Explicitly, we obtain

$$
P=\gamma \dot{q} I_{0}+p Q
$$

where $I_{0}$ is given by Eq. (5.7). Using Eq. (5.15), we can rewrite this as

$$
P=\gamma M_{0} \dot{q}-\frac{\partial U[q, \dot{q}]}{\partial \dot{q}},
$$


which we recognize as identical to the canonical momentum $P_{q}=\frac{\delta L}{\delta \dot{q}}$ given by Eq. (5.28). The Lagrange equation for $P_{q}$ is

$$
\dot{P}_{q}=-\frac{\partial U[q, \dot{q}]}{\partial q}=\frac{d}{d t}\left(M_{0} \gamma \dot{q}-\frac{\partial U[q, \dot{q}]}{\partial \dot{q}}\right) .
$$

\section{STABILITY CONJECTURE}

For the NLSE under the influence of external forces, one could determine fairly accurately the domains of stability of solitary-wave solutions without solving the exact partial differential equations for the given external force but instead studying the behavior of the collective variables in a variational approximation using known solutions of the unforced problem as the trial wave functions. In Refs. [10,12], it was demonstrated that a reliable dynamical stability criterion for the breakup of the solitary wave under external forces was that the solitary wave will be stable if

$$
\frac{\partial p(t)}{\partial \dot{q}(t)}>0 .
$$

Here $p(t)$ is the normalized momentum of the solitary wave $P(t) / M(t)$, where $M=\int d x \Psi^{\star}(x, t) \Psi(x, t)$ is the "mass" of the solitary wave. For the NLDE $Q$ takes the place of $M$. However, $Q$ is a conserved variable so one can use the canonical momentum $P(t)$ instead of $P(q, \dot{q}) / Q$ to study stability. Using our collective coordinate theory, this leads to the criterion that a necessary (but insufficient) condition for stability of the solitary waves of the CC theory is that

$$
\frac{\partial P(q, \dot{q})}{\partial \dot{q}}=\gamma^{3} M_{0}-\frac{\partial^{2} U}{\partial \dot{q}^{2}}>0 .
$$

Note that the right-hand side of Eq. (6.2) plays the role of a time-dependent mass. The sufficient condition for the solitary-wave solution to the $\mathrm{CC}$ equation to be unstable in our simulations is that

$$
\frac{\partial P(q, \dot{q})}{\partial \dot{q}}<0 .
$$

Following Comech's reasoning [15], we expect that, in the nonrelativistic regime where $\omega$ is close to $m$, that this criterion will be valid in determining stability in the case of external sources. However, for the NLDE, we are instead studying the effect of external potentials on solitary-wave motion, which is a different problem. For the external potentials we have studied in this paper, Eq. (6.3) was never satisfied, so the instabilities that we see are instead often related to the $\omega$ instabilities already present in the problem without external potentials or some other cause. Thus, our hope of obtaining a simple way of determining the domain of instabilities using Eq. (6.3) was not borne out.

\section{SIMPLE EXTERNAL POTENTIALS}

\section{A. Simulations}

The numerical simulations have been performed by means of a fourth-order Runge-Kutta method. We choose $N+1$ points starting at $n=0$ and vanishing boundary conditions $\Psi( \pm L, t)=0$. The other parameters related with the discretization of the system are $x \in[-100,100], \Delta x=0.02$,
$\Delta t=0.0001$. For our initial conditions on the solitary wave, we use the exact one-solitary-wave solutions of the unforced nonlinear Dirac equation discussed in Sec. II. Since we would like to compare the exact numerical solution with the solution of the $\mathrm{CC}$ equations, we need to define how we determine the position of the solitary wave. In our numerical computation of $q(t)$, we have used the first moment of the charge, i.e.,

$$
q(t)=Q_{1}(t) / Q(t)
$$

where

$$
Q_{1}=\int d x x \Psi^{\dagger}(x, t) \Psi(x, t), \quad Q=\int d x \Psi^{\dagger}(x, t) \Psi(x, t) .
$$

For the collective variable $P(t)$ we use the definition found in Eq. (5.34). What we will find from our numerical simulations is that the shape of the solitary wave starts deforming once one or both of these collective variables $q(t)$ and $P(t)$ become unsmooth or rapidly varying functions of time. Simultaneously, these variables begin to differ from their counterparts found by solving the $\mathrm{CC}$ equations.

\section{B. Linear potential (ramp potential)}

Consider the constant external force with scalar potential $V(x)=-V_{1} x$ and $V_{1}>0$. We then have from Eq. (5.10) that

$$
U=-V_{1} q(t) Q,
$$

and the force law then becomes

$$
\frac{d}{d t}\left(M_{0} \gamma \dot{q}\right)=V_{1} Q
$$

Integrating once [starting at an initial velocity $\dot{q}(0)$ ] one has

$$
\frac{\dot{q}}{\sqrt{1-\dot{q}^{2}}}=c_{1} t+c_{2}
$$

where $c_{1}=V_{1} Q / M_{0}$ and $c_{2}=\frac{\dot{q}(0)}{\sqrt{1-\dot{q}(0)^{2}}}$. Integrating, we obtain

$$
q(t)=\frac{\sqrt{\left(c_{1} t+c_{2}\right)^{2}+1}}{c_{1}}-\frac{\sqrt{c_{2}^{2}+1}}{c_{1}}+q(0) .
$$

This is the standard result for a relativistic point particle undergoing constant acceleration. If we choose $q(0)=\dot{q}(0)=$ 0 , we get the simpler expression

$$
q(t)=\frac{1}{c_{1}}\left[\sqrt{1+c_{1}^{2} t^{2}}-1\right] .
$$

The energy of the solitary wave is conserved

$$
E=M_{0} \gamma+U=M_{0},
$$

and the force law is now

$$
\dot{P}=-\frac{\partial U}{\partial q}=V_{1} Q,
$$

so

$$
P=V_{1} Q t .
$$

Since $U$ in this case is independent of $\dot{q}$, we find from Eq. (6.2) that

$$
\frac{\partial P}{\partial \dot{q}}=\gamma^{3} M_{0}>0,
$$


thus the necessary condition for stability is fulfilled. In this section and what follows we will confine ourselves to the case where $\kappa=1$ and also $Q=1$. For that case, from the Bogolubsky stability requirement [17] we know that, without forcing when $\omega<\omega_{c}=0.697586$, the solitary waves are unstable.

Let us look at the case where the unforced solitary wave is stable. For $\omega=0.9, g=1$ we have solved numerically the NLDE for $V_{1}=0.01$ (as well as $V_{1}=10^{-3}$ and $V_{1}=10^{-4}$ ). We find, for all these values of $V_{1}$, that the solitary wave is stable at all simulation times and the center of the solitary wave follows the analytic formula we derived from the $\mathrm{CC}$ equation for $q(t)$, namely Eq. (7.7). In Fig. 8 we display the results of the simulation for the charge density $\rho_{Q}\left(x, t^{*}\right)$ and $q(t), P(t)$ for $V_{1}=0.01$. We notice that the width of the solitary wave gets Lorentz contracted as the velocity increases (this effect is not apparent for the smaller values of $V_{1}$ ). Because the charge is conserved, the height of the solitary wave increases due to the increase of $\gamma(t)$.

For the case $\omega=0.3$, the unforced solitary wave has double humped behavior and is unstable at late times. Here our simulations show that until the instability sets in (around $t \approx 110$ for $m=1, g=1, V_{1}=0.01$ ) the position of the solitary wave follows the analytic solution of the CC equation [Eq. (7.7)]. However, the actual shape of the solitary wave becomes asymmetric, with the left hump becoming higher than the right hump as a precursor to the wave becoming unstable. This is shown in Fig. 9, where $\rho_{Q}\left(x, t^{*}\right)$ is plotted against $x$ for various $t=t^{\star}$. In Fig. 10 we give results of the simulation for the case where $\omega=0.3, V_{1}=0.0001$. Here, looking at $q(t)$, we explicitly see that, around $t=120$, the solution of the NLDE diverges from the solution of the CC equation. Also for this value of the potential the solitary wave humps are symmetric and that the single solitary wave breaks up into two solitary waves with some radiation when it goes unstable.

\section{Harmonic potential}

Let us consider the case of an external harmonic potential, $V(x)=\frac{1}{2} V_{2} x^{2}$ and $V_{2}>0$. For that case from Eq. (5.10) we find that

$$
\begin{aligned}
U= & \frac{1}{2} V_{2} \int_{-\infty}^{\infty} d z\left[\left(q+\frac{z}{\gamma}\right)^{2}\left[A^{2}(z)+B^{2}(z)\right]\right] \\
& =\frac{1}{2} V_{2}\left[Q q^{2}(t)+\left(1-\dot{q}^{2}(t)\right) I_{3}\right],
\end{aligned}
$$

where $I_{3}=\int_{-\infty}^{\infty} d z z^{2}\left[A^{2}(z)+B^{2}(z)\right]$. From Eq. (5.19) we have

$$
F_{\mathrm{eff}}[q, \dot{q}]=-V_{2} I_{3} \ddot{q}-V_{2} Q q,
$$

leading to the equation of motion [see Eq. (5.23)],

$$
\frac{d}{d t}\left\{\left(M_{0} \gamma+V_{2} I_{3}\right) \dot{q}\right\}=-V_{2} q(t) Q
$$

This can be rewritten as

$$
\left(M_{0} \gamma^{3}+V_{2} I_{3}\right) \ddot{q}+V_{2} Q q=0 .
$$
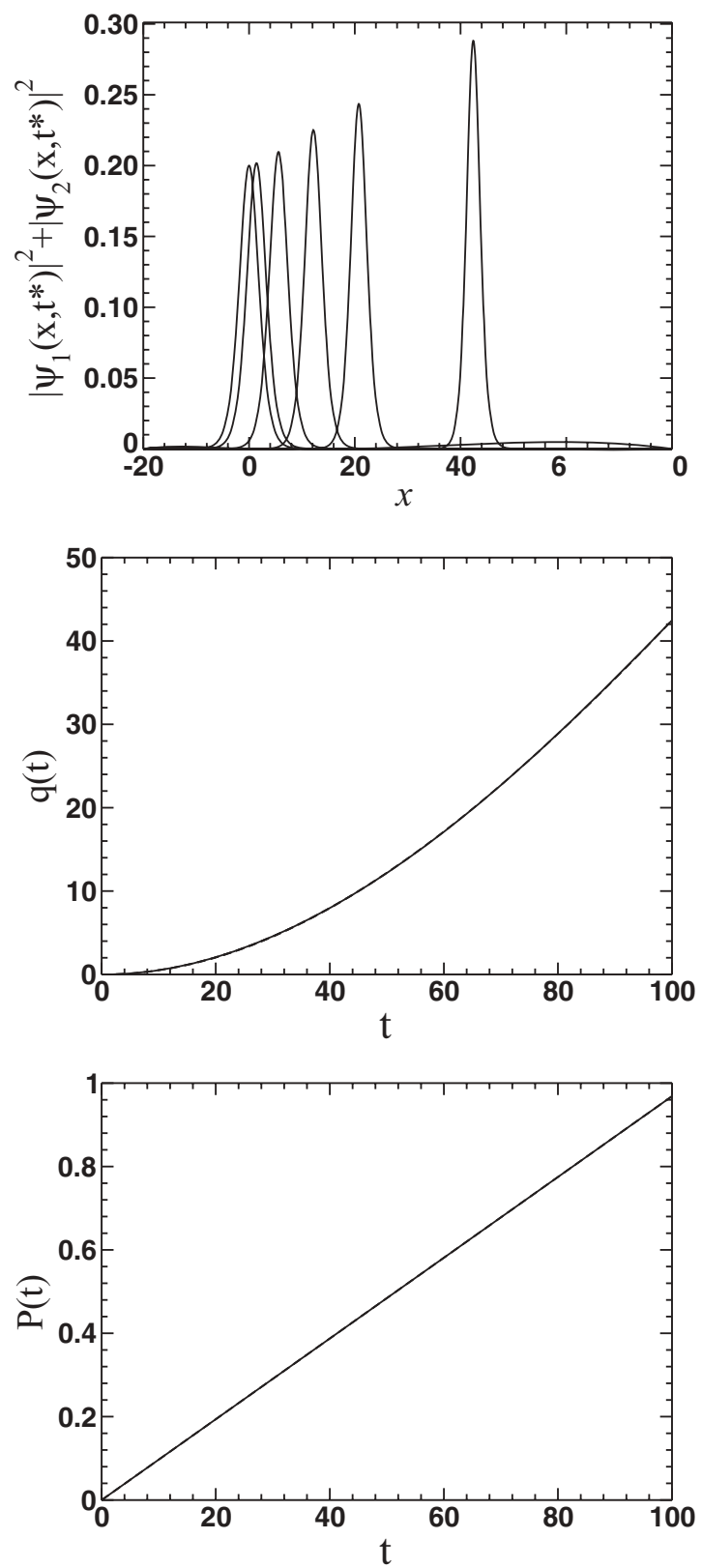

FIG. 8. Simulations with a ramp potential, $V(x)=-V_{1} x$. (Upper panel) Charge density $\rho_{Q}$ at $t^{*}=16.6 ; 33.3 ; 50 ; 66.6 ; 83.3 ; 100$. (Middle and lower panels) Solitary-wave position $q(t)$ and momentum $P(t)$ from analytical results of the CC equations (solid lines) and numerical simulations (dashed lines) of the forced NLDE. The curves are superimposed. For the final time of integration the relative error of $q(t)$ is of order $10^{-5}$. Parameters: $g=1, m=1, \omega=0.9$, and $V_{1}=0.01$. Initial condition: exact solitary wave of the unperturbed NLDE with zero initial velocity.

In the nonrelativistic regime where $\gamma \approx 1$ we recover the oscillator equation for the collective coordinate $q(t)$, namely

$$
\ddot{q}+\Omega^{2} q=0, \quad \Omega^{2}=\frac{V_{2} Q}{M_{0}+V_{2} I_{3}} .
$$

Note that the rest mass is increased by the term $V_{2} I_{3}>0$. For initial conditions $q(0)=0, \dot{q}(0)=v_{0}$ we obtain

$$
q(t)=\frac{v_{0}}{\Omega} \sin \Omega t .
$$



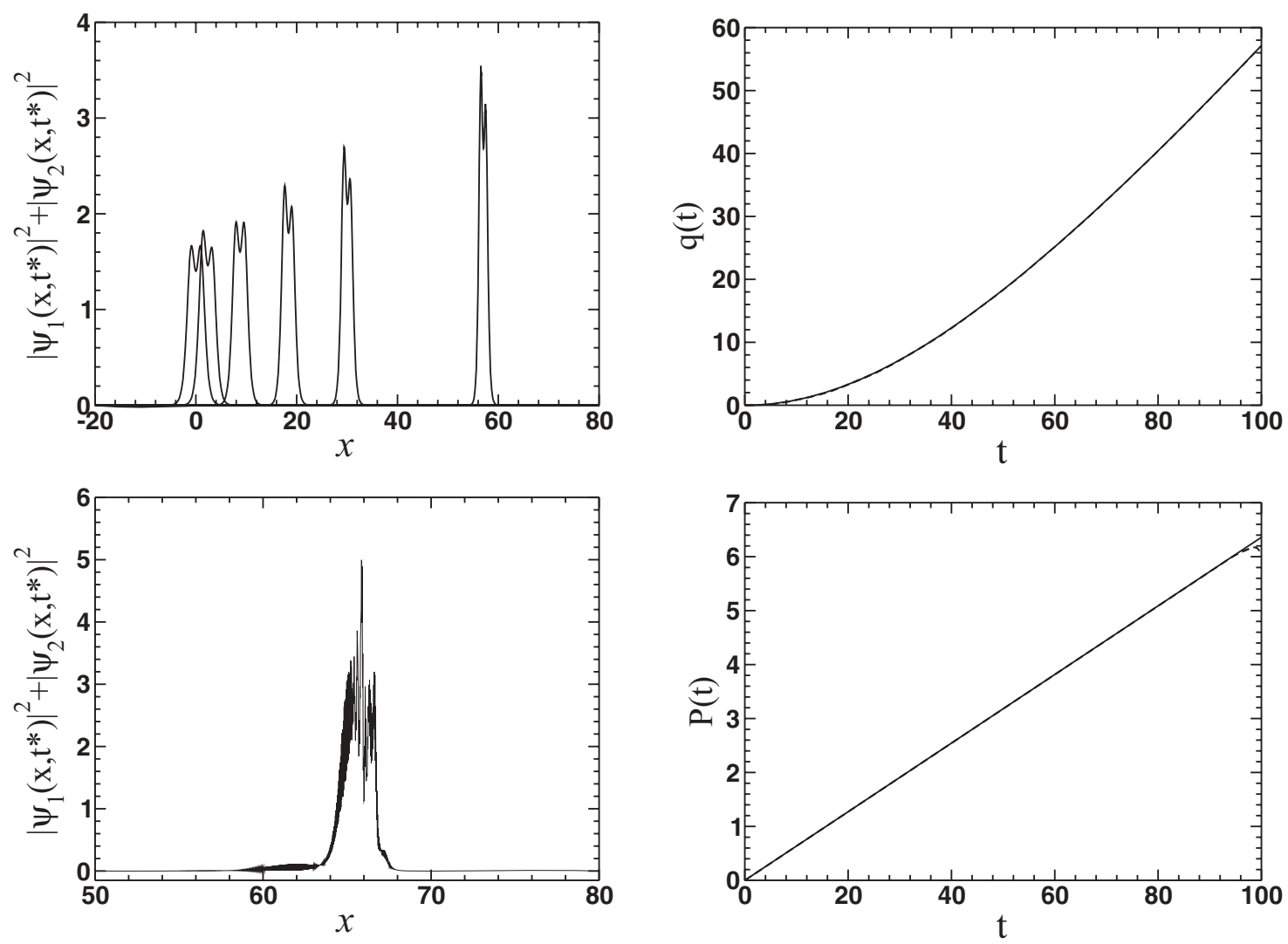

FIG. 9. Simulations with a ramp potential, $V(x)=-V_{1} x$, with $\omega$ in the unstable regime. (Left upper panel) Charge density $\rho_{Q}$ at $t^{*}=16.6 ; 33.3 ; 50 ; 66.6 ; 83.3 ; 100$. (Left lower panel) Charge density at $t^{*}=110$. (Right panels) $q(t)$ and $P(t)$ from analytical results of the $\mathrm{CC}$ equations (solid lines) and from numerical simulations of the forced NLDE (dashed lines); the curves are super-imposed. Parameters: $g=1, m=1, \omega=0.3$, and $V_{1}=0.01$. Initial condition: exact solitary wave of the unperturbed NLDE with zero initial velocity.

\section{Energy conservation}

From the energy conservation equation (5.33) we obtain

$$
E=M_{0} \gamma+\frac{1}{2} Q V_{2} q^{2}+\frac{1}{2} V_{2} I_{3}\left(1+\dot{q}^{2}\right) \text {. }
$$

In the low-velocity limit, we need to keep the first two terms in the expansion of $\gamma$ in the expression for the energy; namely (here we suppress the speed of light in the $v / c$ expansion)

$$
\gamma=1+\frac{1}{2} \dot{q}^{2}+\cdots .
$$

We then have for the solution $q(t)=\frac{v_{0}}{\Omega} \sin \Omega t$ the usual equipartition of energy and that the nonrelativistic energy is twice the initial kinetic energy (apart from a constant),

$$
E=M_{0}+2 T, \quad T=\left(M_{0}+V_{2} I_{3}\right) v_{0}^{2},
$$

with the effective mass of the solitary wave increased over the unforced case by the quantity $V_{2} I_{3}$.

\section{Canonical momentum and stability criterion}

From the equation for the canonical momentum, Eq. (5.36), we find

$$
P=\left(M+V_{2} I_{3}\right) \dot{q}=\left(M_{0} \gamma+V_{2} I_{3}\right) \dot{q}
$$

This again shows the mass increased by $V_{2} I_{3}$. The stability criterion Eq. (6.2) leads to

$$
\frac{\partial P(q, \dot{q})}{\partial \dot{q}}=\gamma^{3} M_{0}+V_{2} I_{3}>0 .
$$

Thus, the necessary condition for stability of the solitary wave is fulfilled.

We would now like to see how well the $\mathrm{CC}$ equations for $q$ and $P$, namely Eqs. (7.15) and (7.21), compare with the numerical solutions of the forced nonlinear Dirac equation. We will choose our initial condition to be $q(0)=0, \dot{q}(0)=v_{0}$ and study both the nonrelativistic regime $\left(v_{0}=0.1\right)$ and the relativistic regime $\left(v_{0}=0.9\right)$. The external potential can be written as

$$
V=\frac{1}{2} V_{2} x^{2}=\frac{1}{2}\left(\frac{x}{l}\right)^{2},
$$

which identifies the characteristic length of the potential as $l=$ $1 / \sqrt{V_{2}}$. We choose the characteristic length of the potential to be large compared to the width of the solitary wave, which is $1 /(2 \beta), \beta=\sqrt{1-\omega^{2}}$. Choosing $V_{2}=10^{-4}$ accomplishes this requirement. At low velocities both $P(t)$ and $\dot{q}(t)$ are proportional to $\cos \Omega t$, thus, $P(\dot{q})$ is a straight line with a positive slope.

First, let us consider the regime where the unforced solitary waves are stable and choose $\omega=0.9, g=1$. In the 

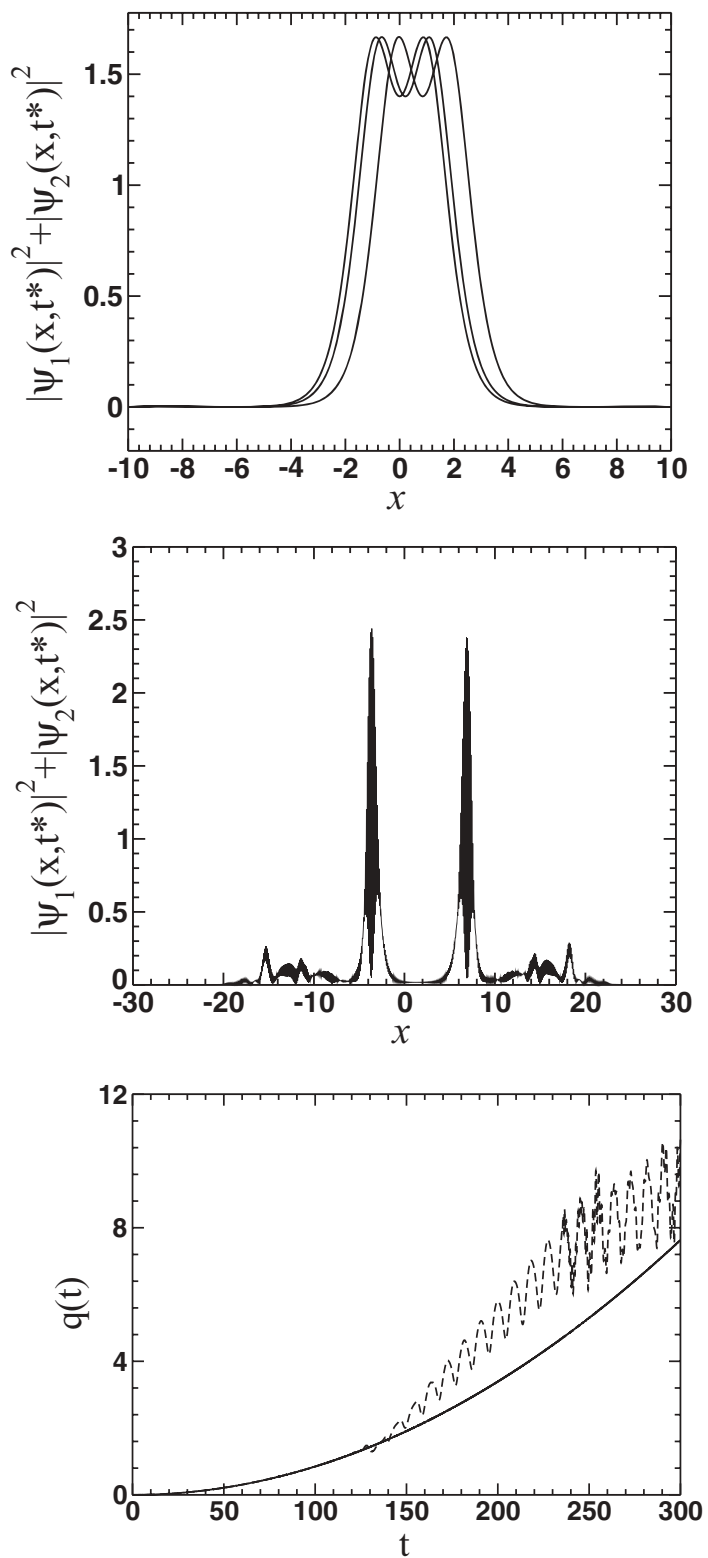

FIG. 10. Simulations with a ramp potential, $V(x)=-V_{1} x$, with $\omega$ in the unstable regime. (Upper panel) Charge density $\rho_{Q}$ at $t^{*}=0 ; 50 ; 100$. (Middle panel) Charge density at $t^{*}=150$. (Lower panel) $q(t)$ from analytical results of the CC equations (solid line) and numerical simulations (dashed line) of the forced NLDE. Parameters: $g=1, m=1, \omega=0.3$, and $V_{1}=0.0001$. Initial condition: exact solitary wave of the unperturbed NLDE with zero initial velocity.

nonrelativistic regime $\left(v_{0}=0.1\right)$ we get the results shown in Fig. 11. The oscillations of both $q(t)$ and $P(t)$ are harmonic as predicted by the $\mathrm{CC}$ equations. The charge density maintains its shape as its position oscillates periodically in time. For $v_{0}=0.9, q$ and $P$ again follow the CC equations for a little less than half the oscillation period but then the solitary wave becomes unstable and the exact simulation of $q$ and $P$ then diverges from the solution to the $\mathrm{CC}$ equations. This is shown in Fig. 12.

Next we consider the regime $0<\omega<\omega_{c}=0.697586$, where the unforced solitary wave is unstable. For the
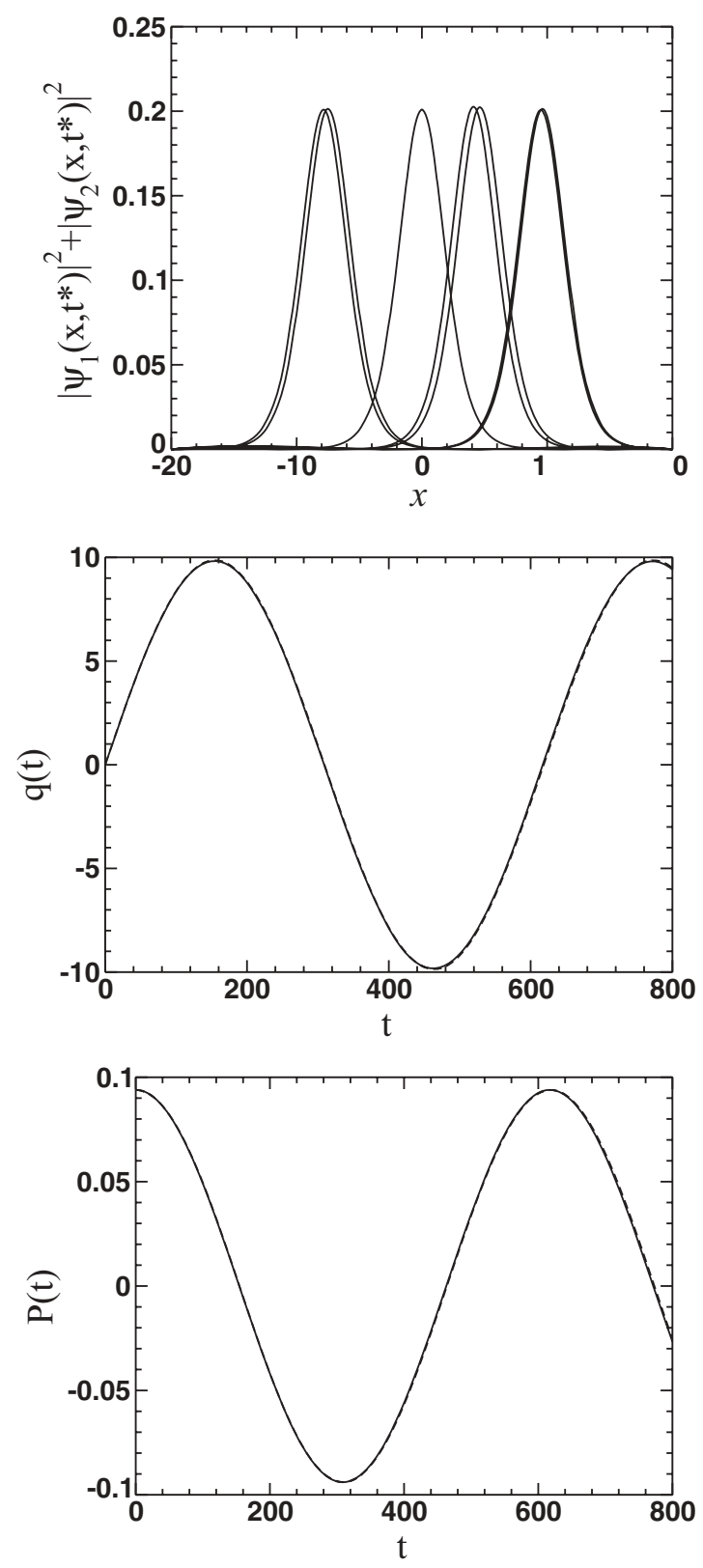

FIG. 11. Harmonic potential, $V(x)=\left(V_{2} / 2\right) x^{2}$. (Upper panel) Charge density $\rho_{Q}$ at $t^{*}=0 ; 133.3 ; 266.6 ; 400 ; 533.3 ; 666.6 ; 800$. (Middle and lower panels) Solitary-wave position $q(t)$ and momentum $P(t)$ from the numerical solutions of the $\mathrm{CC}$ equations (solid lines) and numerical simulations (dashed lines) of the forced NLDE. The curves are superimposed. The charge $Q=0.96864$ and energy $E=0.93921$ are both conserved. Parameters: $g=1, m=1$, $\omega=0.9$, and $V_{2}=10^{-4}$. Initial condition: exact solitary wave of the unperturbed NLDE with initial velocity $v(0)=0.1$.

parameters $\omega=0.3, g=1, V_{2}=10^{-4}$, we obtain the typical result found in the unforced problem that, at around $t=$ 120 , the solitary wave becomes unstable. Until then the CC equations for $q(t)$ and $P(t)$ track the exact solution well. This is seen in Fig. 13. However, the wave function starts becoming asymmetric at late times and departs from our symmetric ansatz even before the solitary wave becomes unstable and breaks into two solitary waves plus some radiation. 

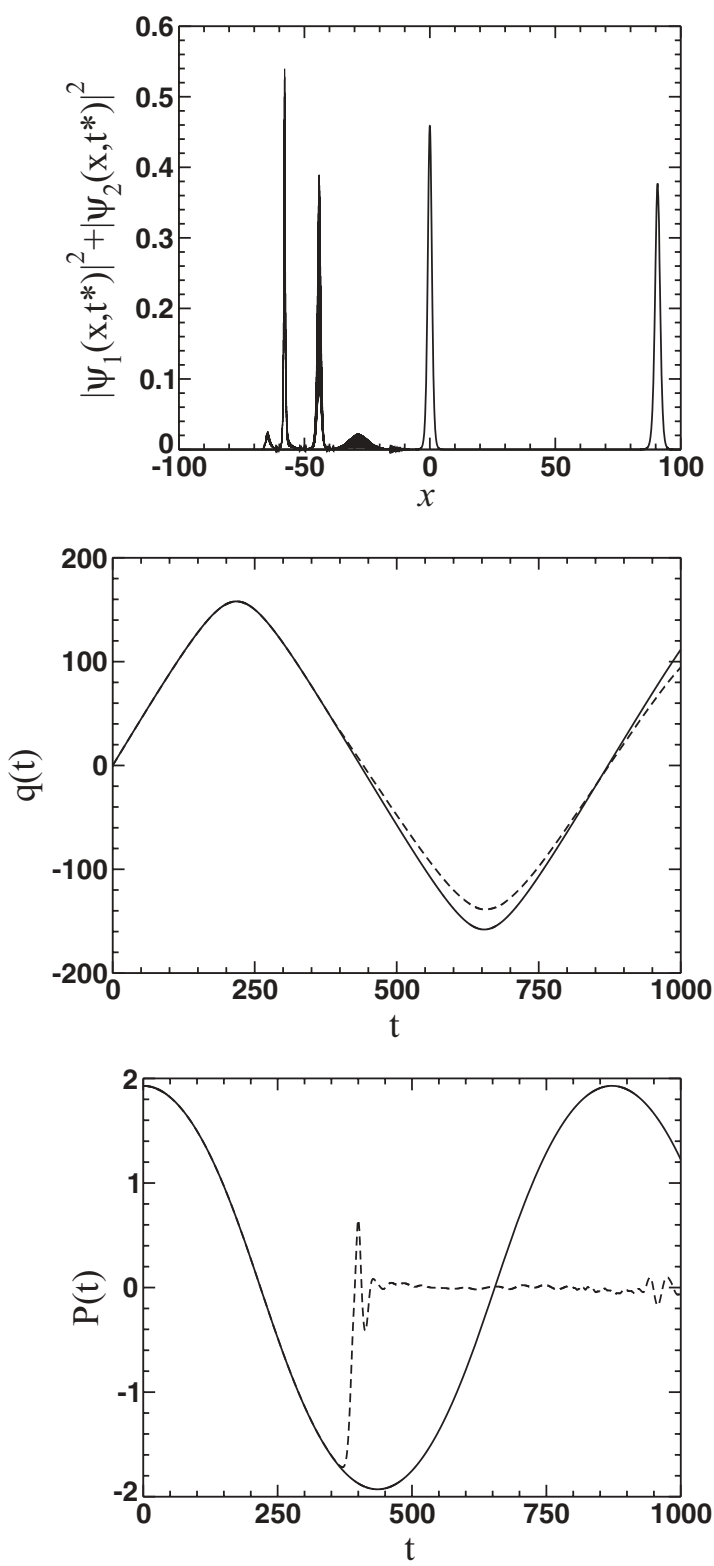

FIG. 12. Harmonic potential, $V(x)=\left(V_{2} / 2\right) x^{2}$. Relativistic regime, unstable solitary wave. (Upper panel) Charge density $\rho_{Q}$ at $t^{*}=0 ; 133.3 ; 500$. (Middle and lower panels) Solitary-wave position $q(t)$ and momentum $P(t)$ from the numerical solutions of the CC equations (solid lines) and numerical simulations (dashed lines) of the forced NLDE. The curves in $P(t)$ are superimposed only till $t=370$. Parameters: $g=1, m=1, \omega=0.9$, and $V_{2}=10^{-4}$. Initial condition: exact solitary wave of the unperturbed NLDE with initial velocity $v(0)=0.9$.

\section{Spatially periodic potentials}

Next consider a spatially periodic potential

$$
V(x)=-\epsilon \cos k x, \quad \epsilon>0,
$$

where the spatial period $L=2 \pi / k \gg 1 / \beta$, and $1 / \beta$ is the width $b$ of the solitary wave. The potential is then a function of $q, \dot{q}$ and is given by

$$
\begin{aligned}
U[q, \dot{q}] & =-\epsilon \cos k q \int d z \cos \frac{k z}{\gamma}\left(A^{2}[z]+B^{2}[z]\right) \\
& =-\epsilon \cos k q I_{4}[\dot{q}],
\end{aligned}
$$

So

$$
\begin{gathered}
\frac{\partial U}{\partial q}=k \epsilon \sin k q I_{4}[\dot{q}] \\
\frac{\partial U}{\partial \dot{q}}=-k \epsilon \gamma \dot{q} \cos k q \int d z z \sin \frac{k z}{\gamma}\left(A^{2}[z]+B^{2}[z]\right) \\
\equiv-k \epsilon \gamma \dot{q} \cos k q I_{5}[\dot{q}] .
\end{gathered}
$$

The generalized force Eq. (5.19) can be written as

$$
\begin{aligned}
F_{\mathrm{eff}}[q, \dot{q}]= & -k \epsilon I_{4}(\dot{q}) \sin (k q(t)) \\
& -k \epsilon \cos (k q) \ddot{q}\left(\gamma^{3} I_{5}[\dot{q}]-k(\gamma \dot{q})^{2} I_{6}[\dot{q}]\right) \\
= & M_{0} \frac{d}{d t}(\gamma \dot{q}),
\end{aligned}
$$

where

$$
I_{6}[\dot{q}]=\int d z z^{2} \cos \frac{k z}{\gamma}\left(A^{2}[z]+B^{2}[z]\right) .
$$

In the nonrelativistic limit $(\dot{q})^{2} \ll 1, \gamma \approx 1$ and we obtain for the force law

$$
\left(M_{0}+k \epsilon I_{5}^{0} \cos k q\right) \ddot{q}+k \epsilon I_{4}^{0} \sin k q=0,
$$

where

$$
\begin{aligned}
& I_{4}^{0}=\int d z \cos k z\left(A^{2}[z]+B^{2}[z]\right) ; \\
& I_{5}^{0}=\int d z z \sin k z\left(A^{2}[z]+B^{2}[z]\right) .
\end{aligned}
$$

When the potential is weak $(\epsilon \ll 1)$, then $k \epsilon I_{5}^{0} \ll M_{0}$ and we obtain the pendulum equation

$$
M_{0} \ddot{q}+k \epsilon I_{4}^{0} \sin k q=0 .
$$

Letting $C=k \epsilon I_{4}^{0} / M_{0}$, the solutions are given by

$$
q(t)=\frac{2}{k} \mathrm{am}\left(\frac{1}{2} \sqrt{k} \sqrt{2 C t^{2}+k c_{1} t^{2}+4 C c_{2} t+2 k c_{1} c_{2} t+2 C c_{2}^{2}+k c_{1} c_{2}^{2}}, \frac{4 C}{2 C+k c_{1}}\right),
$$

where $c_{1}, c_{2}$ are integration constants to be determined by the initial conditions, $q(0)=0 ; \dot{q}(0)=v_{0}$. Here

$$
\operatorname{am}[u, l]=\text { JacobiAmplitude }[u, l],
$$

where the modulus parameter $l$ (usually denoted by $m$ ) is $l=\frac{4 C}{2 C+k c_{1}}$. For the above initial conditions we find

$$
q(t)=\frac{2}{k} \mathrm{am}\left[\frac{k v_{0} t}{2}, \frac{4 C}{k v_{0}^{2}}\right]=\frac{2}{k} \sin ^{-1} \operatorname{sn}\left[\frac{k v_{0} t}{2}, \frac{4 C}{k v_{0}^{2}}\right],
$$



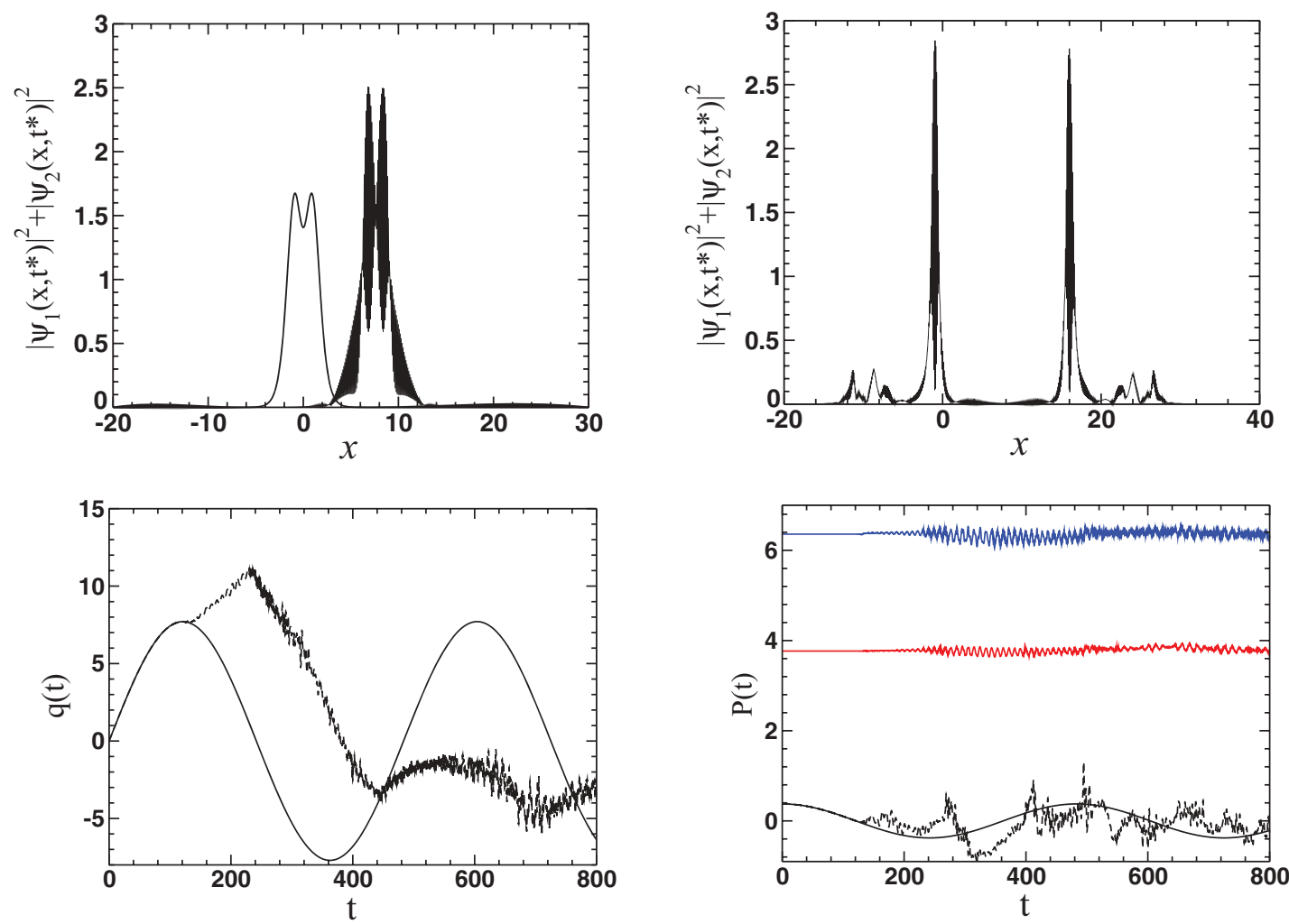

FIG. 13. (Color online) Harmonic potential, $V(x)=\left(V_{2} / 2\right) x^{2}$, with $\omega$ in the unstable regime. (Left upper panel) Charge density $\rho_{Q}$ at $t^{*}=0 ; 133.3$. (Right upper panel) Charge density at $t^{*}=150$. (Lower panels) Solitary-wave position $q(t)$ and momentum $P(t)$ from the numerical solutions of the CC equations (black solid lines) and numerical simulations (dashed lines) of the forced NLDE. The energy (red or middle curve) and charge (blue or upper curve) are also plotted. Parameters: $g=1, m=1, \omega=0.3$, and $V_{2}=10^{-4}$. Initial condition: exact solitary wave of the unperturbed NLDE with initial velocity $v(0)=0.1$.

and $\operatorname{sn}(x, l)$ is a Jacobi elliptic function.

\section{Energy conservation}

From Eq. (5.33) we have that the solitary-wave energy is given by

$$
E=\gamma M_{0}-\epsilon \cos k q I_{4}[\dot{q}]+\epsilon k \gamma \cos k q \dot{q}^{2} I_{5}[\dot{q}] .
$$

In the nonrelativistic limit we obtain

$$
E=M_{0}-\epsilon \cos k q I_{4}^{0}+\left(\frac{M_{0}}{2}+\epsilon k I_{5}^{0} \cos k q\right) \dot{q}^{2} .
$$

In the case of a weak potential (except for $M_{0} \rightarrow 0$ when $\omega \rightarrow 1)$

$$
E=\left(1+\frac{\dot{q}^{2}}{2}\right) M_{0}-\epsilon \cos k q I_{4}^{0} .
$$

\section{Solitary-wave momentum and dynamical stability}

The solitary-wave momentum is given by Eq. (5.36) and becomes

$$
P=\gamma\left(M_{0}+k \epsilon I_{5}[\dot{q}] \cos k q\right) \dot{q} .
$$

In the nonrelativistic regime we obtain

$$
P=\left(M_{0}+k \epsilon I_{5}^{0} \cos k q\right) \dot{q} .
$$

The necessary condition for stability,

$$
\frac{d P}{d \dot{q}}=\left(M_{0}+k \epsilon I_{5}^{0} \cos k q\right)>0,
$$

is satisfied except in the regime where $M_{0} \rightarrow 0$, which is when $\omega \rightarrow 1$. In that regime, the solitary wave is very broad and the condition $2 \pi / k \gg b$ is not fulfilled.

\section{Numerical results for $q(t)$ and $P(t)$}

For the pendulum equation there is a critical initial velocity at which the coordinate $q(t)$ makes a transition from periodic motion to unbounded motion. This occurs when the modulus parameter $l=1$. This yields the condition

$$
v_{c}=\sqrt{\frac{4 I_{4}^{0} \epsilon}{M_{0}} .}
$$

Depending on our choice of parameters, for small-enough $\epsilon$, $v_{c}$ will be in the nonrelativistic regime. We choose $\omega$ to be in the stability region for the unforced problem (see Sec. III). For $g=1, m=1, \omega_{c}=0.697586$ and choosing $\omega=0.9$, then the width of the solitary wave is $1 /(2 \beta)=1.15$. If we choose $k=0.1$, then the characteristic wavelength $2 \pi / k=62.8 \gg$ $1 /(2 \beta)$. From Eqs. (2.47) and (2.48) we have that

$$
\begin{gathered}
Q=0.968644 ; \quad H_{1}=0.0625108=I_{0}, \\
M_{0}=H_{1}+\omega Q=0.934291 .
\end{gathered}
$$



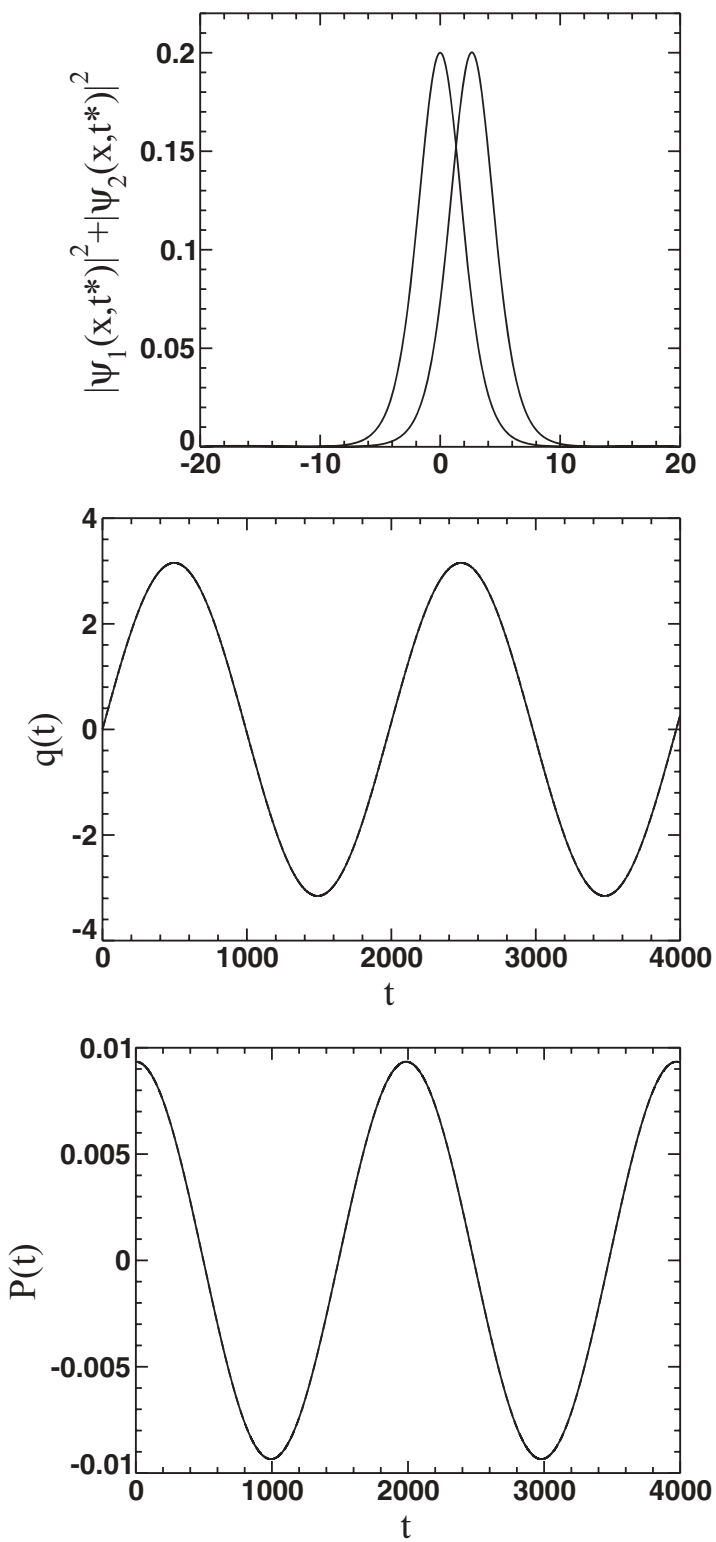

FIG. 14. Periodic potential, $V(x)=-\epsilon \cos (k x)$, very low initial velocity. (Upper panel) Charge density $\rho_{Q}$ at $t^{*}=0 ; 2666.6$. (Middle panel) Solitary-wave position $q(t)$ from the numerical solution of Eq. (7.30) (solid line), approximate analytical expression (7.35) (dotted line), and numerical simulations (dashed line) of the forced NLDE. The three curves are superimposed. (Lower panel) Momentum $P(t)$ from the numerical solutions of Eq. (7.30) (solid line) and numerical simulations (dashed line) of the forced NLDE. The curves are superimposed. Parameters: $g=1, m=1, \omega=0.9, \epsilon=0.001$, and $k=0.1$. Initial condition: exact solitary wave of the unperturbed NLDE with initial velocity $v(0)=0.01$.

The other constants for this initial condition from Eq. (7.31) are

$$
I_{4}^{0}=0.94632 ; \quad I_{5}^{0}=0.433477 .
$$

We have, first, compared the analytical solution Eq. (7.35) of the pendulum equation with the numerical solution of Eq. (7.30). For $\epsilon<0.1$ the results are practically identical, for $\epsilon \geqslant 1$ deviations occur.
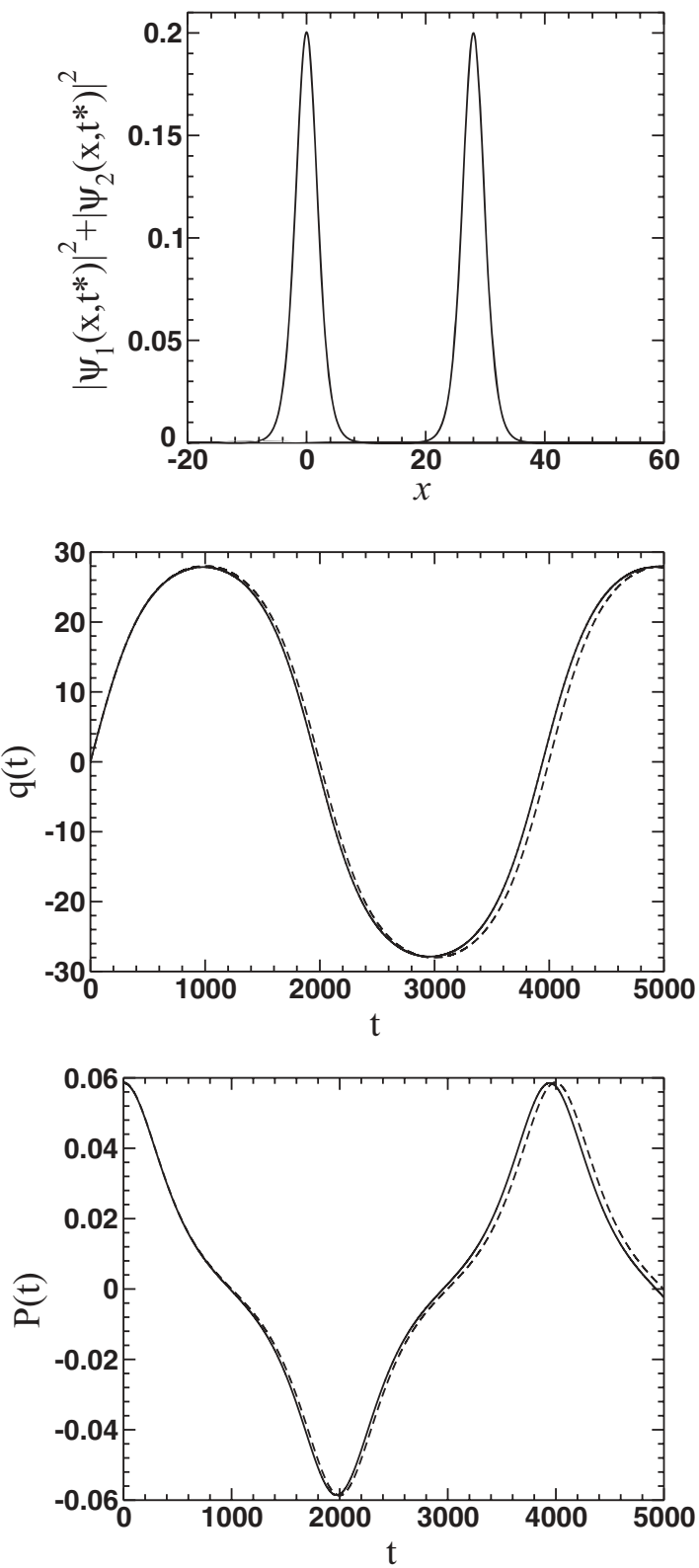

FIG. 15. Spatially periodic potential, $V(x)=-\epsilon \cos (k x)$, initial velocity just below $v_{c}$. (Upper panel) Charge density $\rho_{Q}$ at $t^{*}=0 ; 5000$. (Middle panel) Solitary-wave position $q(t)$ from the numerical solution of Eq. (7.30) (solid line), approximate analytical expression (7.35) (dotted line), and numerical simulations (dashed line) of the forced NLDE. Solid and dotted lines are superimposed. (Lower panel) Momentum $P(t)$ from the numerical solution of Eq. (7.30) (solid line) and numerical simulations (dashed line) of the forced NLDE. Parameters: $g=1, m=1, \omega=0.9, \epsilon=0.001$, and $k=0.1$. Initial condition: exact solitary wave of the unperturbed NLDE with initial velocity $v(0)=0.0626619$.

Specifically we have chosen the initial condition $q(0)=$ $0, \dot{q}=v_{0}$ for the three cases (1) $v_{0} \ll v_{c} \ll 1$ and then $v_{0}$ slightly below (2) and above (3) the critical value $v_{c}$, namely

$$
v_{0}=v_{c} \mp .001 .
$$



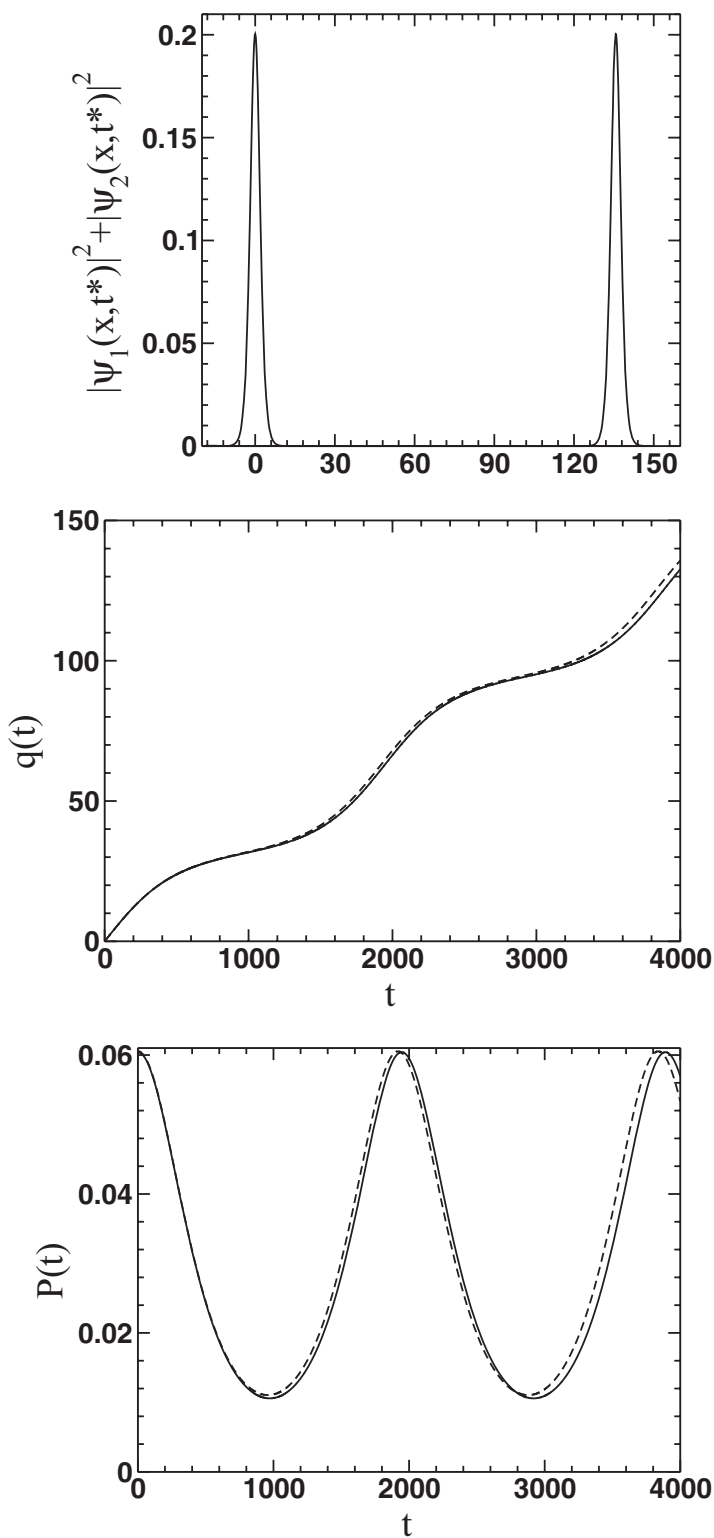

FIG. 16. Spatially periodic potential, $V(x)=-\epsilon \cos (k x)$, initial velocity just above $v_{c}$. (Upper panel) Charge density $\rho_{Q}$ at $t^{*}=0 ; 4000$. (Middle panel) Solitary-wave position $q(t)$ from the numerical solution of Eq. (7.30) (solid line), approximate analytical expression (7.35) (dotted line), and numerical simulations (dashed line) of the forced NLDE. Solid and dotted lines are superimposed. (Lower panel) Momentum $P(t)$ from the numerical solution of Eq. (7.30) (solid line) and from numerical simulations (dashed line) of the forced NLDE. Parameters: $g=1, m=1, \omega=0.9, \epsilon=0.001$, and $k=0.1$. Initial condition: exact solitary wave of the unperturbed NLDE with initial velocity $v(0)=0.0646619$.

Choosing $\epsilon=0.001$ yields $v_{c}=0.0636619$, which is in the nonrelativistic regime, so we expect Eq. (7.35) to hold. In Fig. 14 we show that for $v_{0}=0.01$ the analytic nonrelativistic result and the numerical solution of Eq. (7.30) give the same results as the solution of the NLDE. We also see that the shape of the charge density does not change in time. In Figs. 15 and 16 we show that just below and above the critical velocity, respectively, the analytical result (7.35) agrees with the numerical solution of Eq. (7.30), but both results differ very slightly from the simulation results.

A summary of the result of our simulations of solitary waves in different external fields is presented in Table I.

\section{CONCLUSIONS}

In this study we have found exact solutions to the NLDE with scalar-scalar interactions of the form $\frac{g^{2}}{\kappa+1}(\bar{\Psi} \Psi)^{\kappa+1}$ in certain external fields. We have discussed their stability with respect to " $\omega$ " variations. We have also introduced a collective coordinate method for studying the time evolution of solitary waves in external fields and determined simple equations for the collective coordinates that parallel those of a relativistic point particle. We found that unless (or until) the solitary waves displayed an instability, the collective coordinates describing the position and momentum in the $\mathrm{CC}$ equations gave remarkably good agreement with their counterparts from our simulations. We then presented a generalization of a dynamical stability criterion, based only on solving the $\mathrm{CC}$ equations, that was useful in studying the stability of solitary waves in the forced NLSE problem. For our simulations of the exact evolution, as well as the evolution of the collective coordinates, we concentrated on $\kappa=1$. For the forcing terms we used simple test potentials such as ramp, harmonic, and periodic potentials. In many instances, we found that the instability of the solitary wave solution was related to the metastability of the solitary wave in the absence of external forces and the critical time for breakup was quite close to the time found for the unforced problem.

We had hoped that a generalization of the method used to map out domains of instability in the NLSE using the much simpler solutions of the collective coordinate problem would also work for the NLDE equation. Unfortunately for the problems we studied, we obtained $\frac{d p}{d \dot{q}}>0$, which fulfills a necessary condition for stability, so this method did not give any information about instabilities. What we did find using the collective coordinate approximation was that, starting with exact solutions of the unforced problem, these solitary waves maintained shape in the $\mathrm{CC}$ approximation apart from the parameters becoming functions of time. The collective variables in the simulations, namely $q(t)$ and $P(t)$, were smooth functions for a reasonable period of time, even in the case when the solitary waves were only metastable. When these collective variables began to rapidly oscillate and/or diverge from their values found in the collective coordinate calculation, then that "defined" the onset of the instability. The criterion we used for the onset of instability using the collective coordinates is a sufficient condition and we found no cases where the condition for this dynamic instability was satisfied. Possibly this is a result of the fact that external fields differ from external sources. For the external source problem, we would expect in the nonrelativistic regime that we would recover the known results for the forced NLSE with source terms due to the arguments of Comech [15].

The simulations in this paper are confined to the $\kappa=1$ case. The numerical stability of solitary waves in the absence 
TABLE I. Simulation results for three simple potentials using different parameter sets; $g=1$ and $m=1$.

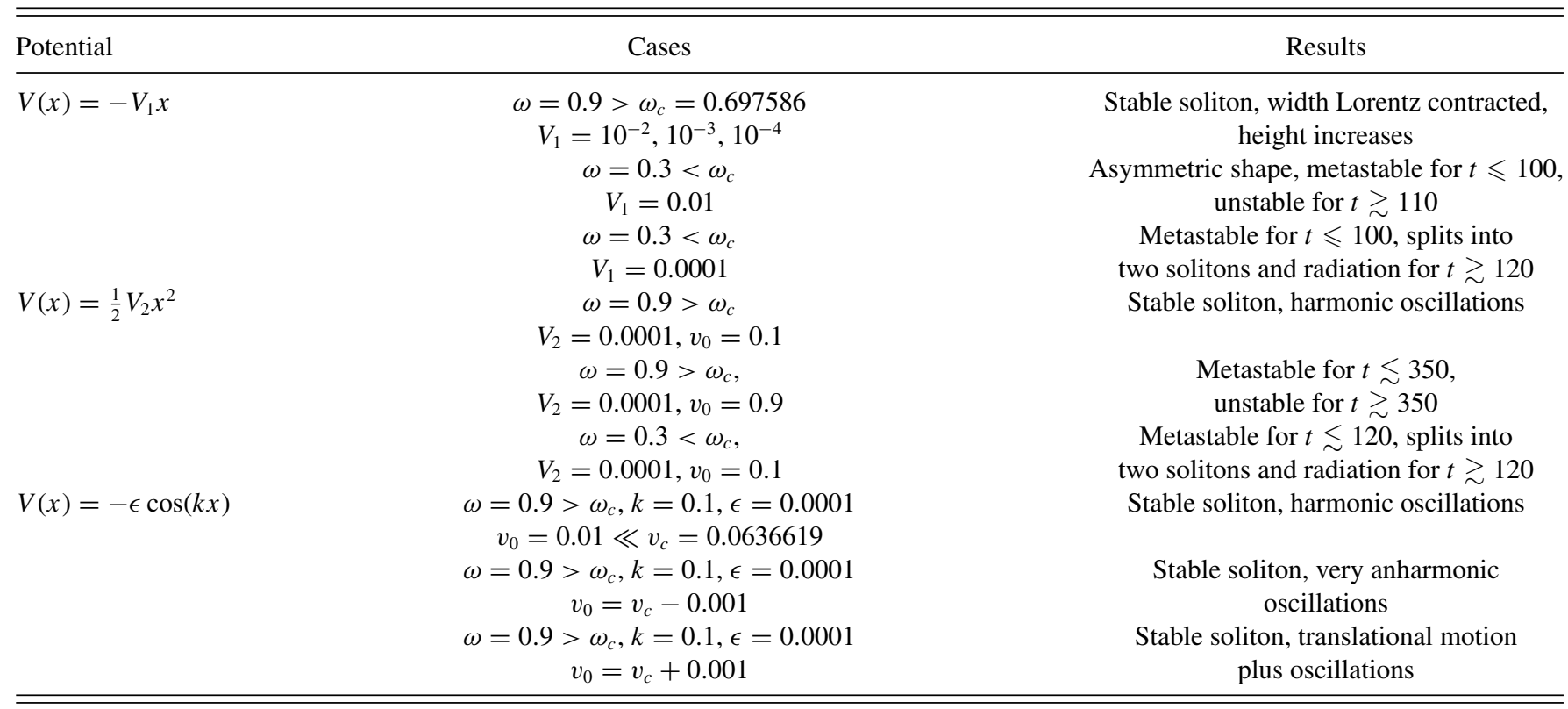

of external potentials for general $\kappa$ will be presented in a subsequent publication [18]. The semiclassical reduction of NLDE to NLSE and implications for solitary-wave stability have been recently discussed in a rigorous fashion by Comech [15]. Our numerical findings [18] agree with his analysis in the nonrelativistic regime.

\section{ACKNOWLEDGMENTS}

This work was supported in part by the US Department of Energy. F.G.M. acknowledges the hospitality of the Mathematical Institute of the University of Seville (IMUS) and of the Theoretical Division and Center for Nonlinear Studies at Los Alamos National Laboratory and financial support by the Plan Propio of the University of Seville and by Junta de Andalucia. N.R.Q. acknowledges financial support from the Humboldt Foundation through Research Fellowship for Experienced Researchers SPA 1146358 STP and by the MICINN through FIS2011-24540 and by Junta de Andalucia under Projects No. FQM207, No. FQM-00481, No. P06-FQM-01735, and No. P09-FQM-4643.

\section{APPENDIX: REST-FRAME IDENTITIES}

In the rest frame, energy-momentum conservation leads to identities among the various integrals that arise. The Lagrangian in the axial gauge is given by

$$
\begin{aligned}
\mathcal{L}= & \frac{i}{2}\left[\bar{\Psi} \gamma^{\mu} \partial_{\mu} \Psi-\partial_{\mu} \bar{\Psi} \gamma^{\mu} \Psi\right]-m \bar{\Psi} \Psi+\frac{g^{2}}{\kappa+1}(\bar{\Psi} \Psi)^{\kappa+1} \\
& -V(x) \Psi^{\dagger} \Psi
\end{aligned}
$$

In the rest frame, the wave function is given by

$$
\Psi_{0}=\psi e^{-i \omega t}=\left[\begin{array}{c}
A(x) \\
i B(x)
\end{array}\right] e^{-i \omega t}
$$

The energy-momentum conservation is given by Eq. (2.6) and leads to two independent equations. The first is

$$
\partial_{0} T^{01}+\partial_{1} T^{11}=0 .
$$

In the rest frame $T^{0 x}=0$, so $T^{x x}=$ const. If the solution goes to zero at infinity, then the constant is zero. We then have the relationship

$$
\begin{aligned}
T^{11} & =\frac{i}{2}\left[\bar{\Psi} \gamma^{1} \partial^{1} \Psi-\partial^{1} \bar{\Psi} \gamma^{1} \Psi\right]+\mathcal{L} \\
& =\omega \psi^{\dagger} \psi-m \bar{\psi} \psi+\frac{g^{2}}{k+1}(\bar{\psi} \psi)^{k+1}-V(x) \psi^{\dagger} \psi=0 .
\end{aligned}
$$

Integrating over space we get the relations

$$
\omega Q-m I_{1}+\frac{g^{2}}{\kappa+1} I_{2}-\int d x \rho(x) V(x)=0 .
$$

The second conservation law is

$$
\partial_{0} T^{00}+\partial_{1} T^{10}=0,
$$

which leads to the conservation of energy. The energy of the solitary wave in the rest frame defines the rest mass $M_{0}$,

$$
E=\int T^{00} d x=M_{0}
$$

We have that

$$
\begin{aligned}
T^{00}= & \frac{i}{2}\left[\bar{\psi} \gamma^{1} \partial_{1} \psi-\partial_{1} \bar{\psi} \gamma^{1} \psi\right] \\
& +m \bar{\psi} \psi-\frac{g^{2}}{k+1}(\bar{\psi} \psi)^{k+1}+V(x) \psi^{\dagger} \psi \\
= & \left(A B_{1}-B A_{1}\right)+m\left(A^{2}-B^{2}\right) \\
& -\frac{g^{2}}{k+1}\left(A^{2}-B^{2}\right)^{\kappa+1}+\left(A^{2}+B^{2}\right) V(x) .
\end{aligned}
$$


Integrating, we obtain

$$
M_{0}=I_{0}+m I_{1}-\frac{g^{2}}{k+1} I_{2}+\int \rho(x) V(x) .
$$

Using the identity of Eq. (A5), we then have, even in the presence of interactions, that

$$
M_{0}=I_{0}+\omega Q \text {. }
$$

[1] R. J. Finkelstein, C. Fronsdal, and P. Kaus, Phys. Rev. 103, 1571 (1956).

[2] U. Enz, Phys. Rev. 131, 1392 (1963).

[3] M. Soler, Phys. Rev. D 1, 2766 (1970).

[4] W. A. Strauss and L. Vazquez, Phys. Rev. D 34, 641 (1986).

[5] S. Y. Lee, T. K. Kuo, and A. Gavrielides, Phys. Rev. D 12, 2249 (1975).

[6] Y. Nogami and F. M. Toyama, Phys. Rev. A 45, 5258 (1992).

[7] D. J. Gross and A. Neveu, Phys. Rev. D 10, 3235 (1974).

[8] W. Thirring, Ann. Phys. 3, 91 (1958).

[9] A. Alvarez and B. Carreras, Phys. Lett. A 86, 327 (1981).

[10] F. Cooper, A. Khare, B. Mihaila, and A. Saxena, Phys. Rev. E 82, 036604 (2010).
[11] N. R. Quintero, F. G. Mertens, and A. R. Bishop, Phys. Rev. E 82, 016606 (2010).

[12] F. G. Mertens, N. R. Quintero, and A. R. Bishop, Phys. Rev. E 81, 016608 (2010).

[13] F. G. Mertens, N. R. Quintero, I. V. Barashenkov, and A. R. Bishop, Phys. Rev. E 84, 026614 (2011).

[14] F. Cooper, A. Khare, N. R. Quintero, F. G. Mertens, and A. Saxena, Phys. Rev. E 85, 046607 (2012).

[15] A. Comech, arXiv:1203.3859, and references therein.

[16] N. G. Vakhitov and A. A. Kolokolov, Radiophys. Quantum Electron. 16, 783 (1973).

[17] I. L. Bogolubsky, Phys. Lett. A 73, 87 (1979).

[18] N. R. Quintero, F. G. Mertens, F. Cooper, A. Khare, and A. Saxena (unpublished). 